\title{
FROBENIUS FUNCTORS OF THE SECOND KIND
}

\author{
S. CAENEPEEL, E. DE GROOT, AND G. MILITARU
}

\begin{abstract}
A pair of adjoint functors $(F, G)$ is called a Frobenius pair of the second type if $G$ is a left adjoint of $\beta F \alpha$ for some category equivalences $\alpha$ and $\beta$. Frobenius ring extensions of the second kind provide examples of Frobenius pairs of the second kind. We study Frobenius pairs of the second kind between categories of modules, comodules, and comodules over a coring. We recover the result that a finitely generated projective Hopf algebra over a commutative ring is always a Frobenius extension of the second kind (cf. [27, [17), and prove that the integral spaces of the Hopf algebra and its dual are isomorphic.
\end{abstract}

\section{INTRODUCTION}

Let $R \rightarrow S$ be a ring homomorphism; it is well-known that the restriction of scalars functor has a left adjoint (the induction functor) and also a right adjoint (the coinduction functor). Morita 24 observed that the left and right adjoint are isomorphic if and only if the morphism $R \rightarrow S$ is Frobenius in the sense of [19]. He calls a pair of functors $(F, G)$ a strongly adjoint pair if $G$ is at the same time a right and left adjoint of $F$. In this case $F$ and $G$ have nice properties: $F$ and $G$ are exact, preserve limits and colimits, and injective and projective objects. Recently, strongly adjoint pairs of functors have been reconsidered under the name Frobenius pairs. In 12], general properties of Frobenius pairs are given, and a characterization is given of Frobenius pairs between categories of (co)modules. In [10] and [3. Frobenius pairs between categories of Doi-Koppinen Hopf modules and entwined modules are studied. In [7, the relation between Frobenius and separability properties is studied.

In this note, we study a weaker version of Frobenius pair: a pair of functors $(F, G)$ is called a Frobenius pair of the second type if $G$ is a right adjoint of $F$, and a left adjoint of $\beta F \alpha$, for some category equivalences $\alpha$ and $\beta$. It turns out that Frobenius pairs of the second type have the same nice properties as the original ones, which we now call Frobenius pairs of the first type. In Section 2, we give a generalization and a new proof of a result of Morita 24], and use this to characterize Frobenius pairs of the second kind between categories of modules over rings. We also show that Frobenius ring extensions of the second kind ([16], 25]) provide examples of Frobenius

1991 Mathematics Subject Classification. 16W30.

Research supported by the bilateral project "Hopf Algebras in Algebra, Topology, Geometry and Physics" of the Flemish and Romanian governments. 
pairs of the second kind. We use this machinery in Section 3 to give a generalization of the Larson-Sweedler result that a finite dimensional Hopf algebra over a field $k$ is a Frobenius extension of $k$ (21]): we show that a finitely generated projective Hopf algebra over a commutative ring is always Frobenius of the second type; as a consequence, we obtain that the integral spaces of the Hopf algebra and its dual are isomorphic.

The results of Section 2 can be dualized to functors between categories of comodules; following Takeuchi's approach [30, we give a coalgebra version of Morita's result, and use this to characterize Frobenius pairs of the second kind between categories of comodules. In the final Section [5 we show that the methods developed in 3] can be adapted easily to decide when the functor forgetting the coaction of a coring is Frobenius of the second kind.

\section{Frobenius Functors of the SECOND Kind}

Let $\mathcal{C}$ and $\mathcal{D}$ be categories, and $F: \mathcal{C} \rightarrow \mathcal{D}$ and $G: \mathcal{D} \rightarrow \mathcal{C}$ covariant functors. Recall that $(F, G)$ is called an adjoint pair of functors if the functors

$$
\operatorname{Hom}_{\mathcal{D}}(\mathrm{F}(\bullet), \bullet) \text { and } \operatorname{Hom}_{\mathcal{C}}(\bullet, \mathrm{G}(\bullet))
$$

are naturally isomorphic. It is well-known (see e.g. 22 or any other introduction to the theory of categories) that this is equivalent to the existence of natural transformations

$$
\eta: 1_{\mathcal{C}} \rightarrow G F \text { and } \varepsilon: F G \rightarrow 1_{\mathcal{D}}
$$

such that

$$
\varepsilon_{F(C)} \circ F\left(\eta_{C}\right)=I_{F(C)} \text { and } G\left(\varepsilon_{D}\right) \circ \eta_{G(D)}=I_{G(D)}
$$

for all $C \in \mathcal{C}$ and $D \in \mathcal{D}$. We say that $G$ is a right adjoint of $F$. A typical example from ring theory is the following: let $i: R \rightarrow S$ be a morphism of rings. The restriction of scalars functor

$$
G:{ }_{S} \mathcal{M} \rightarrow{ }_{R} \mathcal{M}
$$

has a left adjoint, the induction functor

$$
F=S \otimes_{R} \bullet:{ }_{R} \mathcal{M} \rightarrow{ }_{S} \mathcal{M}
$$

and a right adjoint, the coinduction functor

$$
F^{\prime}={ }_{R} \operatorname{Hom}(\mathrm{S}, \bullet):{ }_{\mathrm{R}} \mathcal{M} \rightarrow{ }_{\mathrm{S}} \mathcal{M}
$$

If the induction and coinduction functor are naturally isomorphic, then obviously

$$
S \cong{ }_{R} \operatorname{Hom}(\mathrm{S}, \mathrm{R})
$$

Conversely, if $S$ and ${ }_{R}$ Hom $(\mathrm{S}, \mathrm{R})$ are isomorphic rings, and $S$ is finitely generated projective as a left $R$-module, then we can show that $F$ and $F^{\prime}$ are naturally isomorphic. In other words, $F$ and $F^{\prime}$ are naturally isomorphic if and only if $S / R$ is Frobenius in the sense of Kasch (see [18, 19]). From the uniqueness of the adjoint, it follows also that the induction and coinduction functor are naturally isomorphic if and only if the induction functor is not 
only a left but also a right adjoint of the restriction of scalars functor. Morita 24. calls a pair of functors $(F, G)$ a strongly adjoint pair if $G$ is at the same time a right and left adjoint of $F$ (or, equivalently, $F$ is at the same time a left and right adjoint of $G$ ). In 10, and 12, strongly adjoint pairs were reconsidered and were called Frobenius pairs of functors. We will now generalize this definition.

Definition 1.1. Consider functors $F: \mathcal{C} \rightarrow \mathcal{D}, G: \mathcal{D} \rightarrow \mathcal{C}, \alpha: \mathcal{C} \rightarrow \mathcal{C}$ and $\beta: \mathcal{D} \rightarrow \mathcal{D}$.

$(F, G)$ is called an $(\alpha, \beta)$-Frobenius pair of functors if $G$ is a right adjoint of $F$ and a left adjoint of $\beta F \alpha$.

$A\left(1_{\mathcal{C}}, 1_{\mathcal{D}}\right)$-Frobenius pair is called a Frobenius pair of the first kind, or a Frobenius pair of Functors.

An $(\alpha, \beta)$-Frobenius pair, with $\alpha$ and $\beta$ equivalences of categories, is called a Frobenius pair of the second kind.

If $(F, G)$ is an adjoint pair, then $F$ preserves coproducts, colimits, initial objects and cokernels, and $G$ preserves products, limits, final objects and kernels. Therefore, if $(F, G)$ is an adjoint pair between two abelian categories, then $F$ is right exact and $G$ is left exact (see for example [1, I.7.1]). If, moreover, $G$ is right exact, then $F$ preserves projectives. If $F$ is left exact, then $G$ preserves injectives. If $(F, G)$ is a Frobenius pair of the second kind, then $F$ and $G$ are at the same time the left and the right adjoint in an adjoint pair, and we conclude:

Proposition 1.2. Let $(F, G)$ be a Frobenius pair of the second kind between two categories $\mathcal{C}$ and $\mathcal{D}$.

1) $F$ and $G$ preserve limits and colimits, and, in particular, products and coproducts, kernels and cokernels, initial and final objects.

2) If $\mathcal{C}$ and $\mathcal{D}$ are abelian categories, then $F$ and $G$ are exact, and preserve injective and projective objects.

\section{Modules OVER RINGS}

Let $R$ and $S$ be rings, and consider bimodules $\Lambda \in{ }_{S} \mathcal{M}_{R}$ and $X \in{ }_{R} \mathcal{M}_{S}$. Then we can define four other bimodules, in the following fashion

$$
\begin{array}{cc}
\Lambda^{*}=\operatorname{Hom}_{\mathrm{R}}(\Lambda, \mathrm{R}) \in \mathrm{R}_{\mathrm{R}} \mathcal{M}_{\mathrm{S}} & (r \varphi s)(\lambda)=r \varphi(s \lambda) \\
{ }^{*} \Lambda={ }_{S} \operatorname{Hom}(\Lambda, \mathrm{S}) \in{ }_{\mathrm{R}} \mathcal{M}_{\mathrm{S}} & (\lambda)(r \varphi s)=((\lambda r) \varphi) s \\
\operatorname{Hom}_{\mathrm{R}}(\Lambda, \Lambda) \in \mathcal{M}_{\mathrm{S}} & \left(s \varphi s^{\prime}\right)(\lambda)=s \varphi\left(s^{\prime} \lambda\right) \\
{ }_{S} \operatorname{Hom}(\Lambda, \Lambda) \in \mathrm{R}_{\mathrm{R}} \mathcal{M}_{\mathrm{R}} & (\lambda)\left(r \varphi r^{\prime}\right)=((\lambda r) \varphi) r^{\prime}
\end{array}
$$

The induction functor

$$
F=\Lambda \otimes_{R} \bullet:{ }_{R} \mathcal{M} \rightarrow{ }_{S} \mathcal{M}, F(M)=\Lambda \otimes_{R} M
$$

has a right adjoint, the coinduction functor

$$
G={ }_{S} \operatorname{Hom}(\Lambda, \bullet):{ }_{\mathrm{S}} \mathcal{M} \rightarrow{ }_{\mathrm{R}} \mathcal{M}, \mathrm{G}(\mathrm{N})={ }_{\mathrm{S}} \operatorname{Hom}(\Lambda, \mathrm{N})
$$


with the left $R$-action on $G(N)={ }_{S} \operatorname{Hom}(\Lambda, \mathrm{N})$ given by

$$
(\lambda)(r f)=(\lambda r) f
$$

for all $\lambda \in \Lambda, r \in R$ and $f \in{ }_{S} \operatorname{Hom}(\Lambda, \mathrm{N})$. The unit and counit of the adjunction are

$$
\begin{gathered}
\eta_{M}: M \rightarrow G F(M)={ }_{S} \operatorname{Hom}\left(\Lambda, \Lambda \otimes_{\mathrm{R}} \mathrm{M}\right) ;(\lambda)\left(\eta_{\mathrm{M}}(\mathrm{m})\right)=\lambda \otimes \mathrm{m} \\
\varepsilon_{N}: F G(N)=\Lambda \otimes_{R}{ }_{S} \operatorname{Hom}(\Lambda, \mathrm{N}) \rightarrow \mathrm{N} ; \varepsilon_{\mathrm{N}}(\lambda \otimes \mathrm{f})=(\lambda) \mathrm{f}
\end{gathered}
$$

The converse also holds: if $(F, G)$ is an adjoint pair between ${ }_{R} \mathcal{M}$ and ${ }_{S} \mathcal{M}$, then $F$ and $G$ are additive (see 1 , I.7.2]). $F$ has a right adjoint, and preserves therefore cokernels and arbitrary coproducts (see [1, I.7.1]), and from the Eilenberg-Watts Theorem (see [1, II.2.3]), it follows that $F \cong \Lambda \otimes_{R} \bullet$ for some $\Lambda \in{ }_{S} \mathcal{M}_{R}$. From the uniqueness of the adjoint, if follows that $G \cong$ ${ }_{S} \operatorname{Hom}(\Lambda, \bullet)$.

We also consider the functor

$$
G_{1}:{ }_{S} \mathcal{M} \rightarrow{ }_{R} \mathcal{M} ; G_{1}(N)={ }^{*} \Lambda \otimes_{S} N
$$

We have a natural transformation $\gamma: G_{1} \rightarrow G$ given by

$$
\gamma_{N}:{ }^{*} \Lambda \otimes_{S} N \rightarrow{ }_{S} \operatorname{Hom}(\Lambda, \mathrm{N}) ;(\lambda)\left(\gamma_{\mathrm{N}}(\mathrm{f} \otimes \mathrm{n})\right)=(\lambda) \mathrm{fn}
$$

If $\Lambda$ is finitely generated and projective as a left $S$-module, then $\gamma$ is a natural isomorphism.

Now consider the functor

$$
G_{2}:{ }_{S} \mathcal{M} \rightarrow{ }_{R} \mathcal{M} ; G_{2}(N)=X \otimes_{S} N
$$

When is $\left(F, G_{2}\right)$ an adjoint pair? From the uniqueness of the adjoint, we can see that an equivalent question is: when is $G \cong G_{2}$ ? Or, in other words, when is the induction functor $G_{2}$ representable? The clue to the answer is the following Lemma, where we also consider the functors

$$
G_{2}^{\prime}=\bullet \otimes_{S} \Lambda: \mathcal{M}_{S} \rightarrow \mathcal{M}_{R} \text { and } F^{\prime}=\bullet \otimes_{R} X: \mathcal{M}_{R} \rightarrow \mathcal{M}_{S}
$$

We use the following notation: for two functors $F, G: \mathcal{C} \rightarrow \mathcal{D}$. $\underline{\underline{\operatorname{Nat}}}(F, G)$ is the class consisting of all natural transformations from $F$ to $\bar{G}$. For a commutative ring, and an $R$-bimodule $M, C_{R}(M)=\{m \in R \mid \mathrm{rm}=$ $m r$, for all $r \in R\}$.

Lemma 2.1. Let $R, S, \Lambda, X, F, G, G_{1}, G_{2}$ be as above. Then we have isomorphisms

$$
\begin{aligned}
& \underline{\underline{\mathrm{Nat}}}\left(1_{R} \mathcal{M}, G_{2} F\right) \cong \underline{\underline{\operatorname{Nat}}}\left(1_{\mathcal{M}_{R}}, G_{2}^{\prime} F^{\prime}\right) \\
& \cong{ }_{R} \mathrm{Hom}_{\mathrm{R}}\left(\mathrm{R}, \mathrm{X} \otimes_{\mathrm{S}} \Lambda\right) \cong \mathrm{C}_{\mathrm{R}}\left(\mathrm{X} \otimes_{\mathrm{S}} \Lambda\right) \\
& =\left\{\sum_{i} x_{i} \otimes_{S} \lambda_{i} \in X \otimes_{S} \Lambda \mid\right. \\
& \left.\quad \sum_{i} r x_{i} \otimes_{S} \lambda_{i}=\sum_{i} x_{i} \otimes_{S} \lambda_{i} r \text { for all } r \in R\right\}
\end{aligned}
$$




$$
\begin{aligned}
& \underline{\underline{\operatorname{Nat}}}\left(F G_{2}, 1_{S} \mathcal{M}\right) \cong \underline{\underline{\operatorname{Nat}}}\left(F^{\prime} G_{2}^{\prime}, 1_{\mathcal{M}_{S}}\right) \\
& \cong{ }_{S} \operatorname{Hom}_{\mathrm{S}}\left(\Lambda \otimes_{\mathrm{R}} \mathrm{X}, \mathrm{S}\right) \cong{ }_{\mathrm{R}} \operatorname{Hom}_{\mathrm{S}}\left(\mathrm{X},{ }_{\mathrm{S}} \operatorname{Hom}(\Lambda, \mathrm{S})\right)
\end{aligned}
$$

Proof. For a natural transformation $\alpha: 1_{R} \mathcal{M} \rightarrow G_{2} F$, consider $\sum_{i} x_{i} \otimes_{S}$ $\lambda_{i}=\alpha_{R}\left(1_{R}\right)$. The naturality of $\alpha$ implies that $\sum_{i} x_{i} \otimes_{S} \lambda_{i} \in C_{R}\left(X \otimes_{S} \Lambda\right)$. Conversely, given $\sum_{i} x_{i} \otimes_{S} \lambda_{i} \in C_{R}\left(X \otimes_{S} \Lambda\right)$, we consider $\alpha \in \underline{\underline{\operatorname{Nat}}}\left(1_{\mathcal{M}_{R}}, G_{2} F\right)$ given by

$$
\alpha_{M}: M \rightarrow G_{2} F(M)=X \otimes_{S} \Lambda \otimes_{R} M, \alpha_{M}(m)=\sum_{i} x_{i} \otimes_{S} \lambda_{i} \otimes_{R} m
$$

Given a natural transformation $\beta: F G_{2}=\Lambda \otimes_{R} X \otimes_{S} \bullet \rightarrow 1_{S} \mathcal{M}$, we take

$$
\widetilde{\beta}=\beta_{S}: \Lambda \otimes_{R} X \rightarrow S
$$

$\widetilde{\beta}$ is right $S$-linear because $\beta$ is natural. Conversely, given an $S$-bimodule map $\widetilde{\beta}: \Lambda \otimes_{R} X \rightarrow S$, we define a natural transformation $\beta$ by

$$
\beta_{N}: \Lambda \otimes_{R} X \otimes_{S} N \rightarrow N ; \beta_{N}=\widetilde{\beta} \otimes_{S} I_{N}
$$

The $(R, S)$-bimodule map $\Delta: X \rightarrow{ }_{S} \operatorname{Hom}(\Lambda, S)$ corresponding to an $(S, S)$ bimodule map $\widetilde{\beta}: \Lambda \otimes_{R} X \rightarrow S$ is given by

$$
\Delta(x)(\lambda)=\widetilde{\beta}(\lambda \otimes x)
$$

The following is an extended version of [24, Theorem 2.1]. We include a very short proof, based on Lemma 2.1

Theorem 2.2. Let $R$ and $S$ be rings, $\Lambda \in{ }_{S} \mathcal{M}_{R}$ and $X \in{ }_{R} \mathcal{M}_{S}$. Then the following are equivalent.

i) $\left(F=\Lambda \otimes_{R} \bullet, G_{2}=X \otimes_{S} \bullet\right):{ }_{R} \mathcal{M} \rightarrow{ }_{S} \mathcal{M}$ is an adjoint pair of functors;

ii) $\left(F^{\prime}=\bullet \otimes_{R} X, G_{2}^{\prime}=\bullet \otimes_{S} \Lambda\right): \mathcal{M}_{R} \rightarrow \mathcal{M}_{S}$ is an adjoint pair of functors;

iii) $G={ }_{S} \operatorname{Hom}(\Lambda, \bullet)$ and $G_{2}=X \otimes_{S} \bullet$ are naturally isomorphic;

iv) $G^{\prime}=\operatorname{Hom}_{\mathrm{S}}(\mathrm{X}, \bullet)$ and $G_{2}^{\prime}=\bullet \otimes_{S} \Lambda$ are naturally isomorphic;

v) $\Lambda$ is finitely generated projective as a left $S$-module, and

$$
X \cong{ }_{S} \operatorname{Hom}(\Lambda, \mathrm{S}) \text { in }{ }_{\mathrm{R}} \mathcal{M}_{\mathrm{S}}
$$

vi) $X$ is finitely generated projective as a right $S$-module, and

$$
\Lambda \cong \operatorname{Hom}_{\mathrm{S}}(\mathrm{X}, \mathrm{S}) \text { in } \mathrm{S}_{\mathrm{R}}
$$

vii) there exists $z=\sum_{i} x_{i} \otimes_{S} \lambda_{i} \in C_{R}\left(X \otimes_{S} \Lambda\right)$ and $\omega: \Lambda \otimes_{R} X \rightarrow S$ in ${ }_{S} \mathcal{M}_{S}$ such that

$$
\begin{aligned}
\lambda & =\sum_{i} \omega\left(\lambda \otimes x_{i}\right) \lambda_{i} \\
x & =\sum_{i} x_{i} \omega\left(\lambda_{i} \otimes x\right)
\end{aligned}
$$

for all $x \in X$ and $\lambda \in \Lambda$; 
viii) the same condition as vii), but with $z=\sum_{i} x_{i} \otimes_{S} \lambda_{i} \in X \otimes_{S} \Lambda$;

ix) there exist $\Delta: R \rightarrow X \otimes_{S} \Lambda$ in ${ }_{R} \mathcal{M}_{R}$ and $\varepsilon: X \rightarrow{ }_{S} \operatorname{Hom}(\Lambda, S)$ in ${ }_{R} \mathcal{M}_{S}$ such that, with $\Delta\left(1_{R}\right)=\sum x_{i} \otimes \lambda_{i}$,

$$
\begin{aligned}
\lambda & =\sum_{i}(\lambda)\left(\varepsilon\left(x_{i}\right)\right) \lambda_{i} \\
x & =\sum_{i} x_{i}\left(\lambda_{i}\right)(\varepsilon(x))
\end{aligned}
$$

for all $x \in X$ and $\lambda \in \Lambda$;

$\mathrm{x})$ there exist $\Delta: R \rightarrow X \otimes_{S} \Lambda$ in ${ }_{R} \mathcal{M}_{R}$ and $\varepsilon^{\prime}: \Lambda \rightarrow \operatorname{Hom}_{\mathrm{S}}(\mathrm{X}, \mathrm{S})$ in ${ }_{S} \mathcal{M}_{R}$ such that, with $\Delta\left(1_{R}\right)=\sum x_{i} \otimes \lambda_{i}$,

$$
\begin{aligned}
\lambda & =\sum_{i} \varepsilon^{\prime}(\lambda)\left(x_{i}\right) \lambda_{i} \\
x & =\sum_{i} x_{i} \varepsilon^{\prime}\left(\lambda_{i}\right)\left(x_{i}\right)
\end{aligned}
$$

for all $x \in X$ and $\lambda \in \Lambda$;

$\mathrm{xi})$ the same as $i x)$, but we require that $\varepsilon$ is an isomorphism;

xii) the same as $x$ ), but we require that $\varepsilon^{\prime}$ is an isomorphism;

xiii) there exists $\sum_{i} x_{i} \otimes_{S} \lambda_{i} \in C_{R}\left(X \otimes_{S} \Lambda\right)$ such that the map

$$
\widetilde{\varepsilon}:{ }_{S} \operatorname{Hom}(\Lambda, S) \rightarrow \mathrm{X}, \widetilde{\varepsilon}(\mathrm{g})=\sum_{\mathrm{i}} \mathrm{x}_{\mathrm{i}}\left(\left(\lambda_{\mathrm{i}}\right) \mathrm{g}\right)
$$

is an isomorphism in ${ }_{R} \mathcal{M}_{S}$, and the following condition holds for $\lambda, \lambda^{\prime} \in \Lambda$ :

$$
g(\lambda)=g\left(\lambda^{\prime}\right), \text { for all } g \in{ }_{S} \operatorname{Hom}(\Lambda, S) \Longrightarrow \lambda=\lambda^{\prime}
$$

xiv) there exists $\sum_{i} x_{i} \otimes_{S} \lambda_{i} \in C_{R}\left(X \otimes_{S} \Lambda\right)$ such that the map

$$
\widetilde{\varepsilon}^{\prime}: \operatorname{Hom}_{\mathrm{S}}(\mathrm{X}, \mathrm{S}) \rightarrow \Lambda, \widetilde{\varepsilon}^{\prime}(\mathrm{g})=\sum_{\mathrm{i}} \mathrm{g}\left(\mathrm{x}_{\mathrm{i}}\right) \lambda_{\mathrm{i}}
$$

is an isomorphism in ${ }_{R} \mathcal{M}_{S}$, and the following condition holds for $x, x^{\prime} \in X$ :

$$
g(x)=g\left(x^{\prime}\right), \text { for all } g \in \operatorname{Hom}_{\mathrm{S}}(\mathrm{X}, \mathrm{S}) \Longrightarrow \mathrm{x}=\mathrm{x}^{\prime}
$$

Proof. i) $\Rightarrow$ vii). Let $\left(F, G_{2}\right)$ be an adjoint pair, and let $\eta: 1_{R} \mathcal{M} \rightarrow G_{2} F$ and $\eta: F G_{2} \rightarrow 1_{S} \mathcal{M}$ be the unit and counit of the adjunction, and take $\sum_{i} x_{i} \otimes_{S} \lambda_{i}$ as in Lemma 2.1. Equations (4) and (5) follow immediately from equation (11).

i) $\Leftrightarrow$ iii) follows from the uniqueness of adjoints.

vii) $\Rightarrow$ i) . The natural transformations $\eta$ and $\varepsilon$ corresponding to $\sum_{i} x_{i} \otimes \lambda_{i}$ and $\omega$ are the unit and counit of the adjunction.

iii) $\Rightarrow \mathrm{v})$. Equation (4) tells us that $\left\{\lambda_{i}, \omega\left(\bullet \otimes_{R} x_{i}\right)\right\}$ is a finite dual basis for $\Lambda$ as a left $S$-module.

Let $\gamma_{2}: G_{2} \rightarrow G$ be a natural isomorphism. Obviously $\gamma=\gamma_{2, S}: X \rightarrow$ ${ }_{S} \operatorname{Hom}(\Lambda, S)$ is an isomorphism in ${ }_{R} \mathcal{M}$, and we are done if we can show that 
$\gamma$ is right $S$-linear. This follows essentially from the naturality of $\gamma$. For any $t \in S$, we consider the map $f_{t}: S \rightarrow S, f_{t}(s)=s t . f_{t} \in{ }_{S} \mathcal{M}$, and we have a commutative diagram

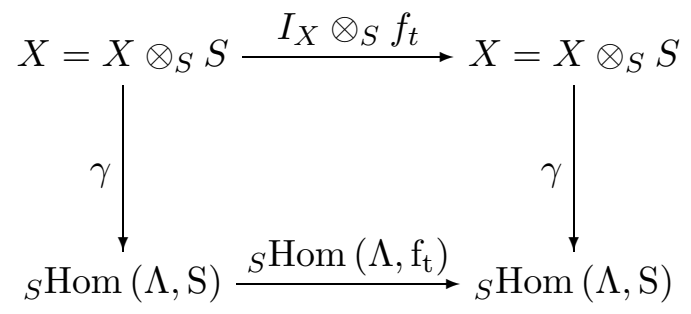

Now observe that $\left(I_{X} \otimes_{S} f_{t}\right)(x)=x t$, and ${ }_{S} \operatorname{Hom}\left(\Lambda, \mathrm{f}_{\mathrm{t}}\right)=\mathrm{f}_{\mathrm{t}} \circ-$, and the commutativity of the diagram implies that

$$
(\lambda)(\gamma(x t))=(\lambda)\left(f_{t} \circ \gamma(x)\right)=((\lambda)(\gamma(x) t)=(\lambda) \gamma(x t)
$$

and $\gamma$ is right $S$-linear.

v) $\Rightarrow$ iii). $\Lambda$ is finitely generated and projective as a left $S$-module, so we have a natural isomorphism

$$
\gamma:{ }_{S} \operatorname{Hom}(\Lambda, \mathrm{S}) \otimes \bullet \rightarrow \mathrm{s}_{\mathrm{S}} \operatorname{Hom}(\Lambda, \bullet)
$$

and from v) it also follows that

$$
{ }_{S} \operatorname{Hom}(\Lambda, \mathrm{S}) \otimes \bullet \cong \mathrm{X} \otimes_{\mathrm{S}} \bullet
$$

vii) $\Rightarrow$ viii) is trivial.

$\underline{\text { viii } \Rightarrow \text { vii) }}$. For all $r \in R$, we have

$$
\begin{aligned}
r z & =\sum_{i} r x_{i} \otimes_{S} \lambda_{i} \\
& =\sum_{i, j} x_{j} \omega\left(\lambda_{j} \otimes_{R} r x_{i}\right) \otimes_{S} \lambda_{i} \\
& =\sum_{i, j} x_{j} \otimes_{S} \omega\left(\lambda_{j} r \otimes_{R} x_{i}\right) \lambda_{i} \\
\text { (41) } & =\sum_{j} x_{j} \otimes_{S} \lambda_{j} r=z r
\end{aligned}
$$

vii) $\Rightarrow$ ix $). \quad \varepsilon$ is defined by $(\lambda)(\varepsilon(x))=\omega\left(\lambda \otimes_{S} x\right)$. It is easy to show that $\varepsilon$ is left $R$-linear and right $S$-linear, and equations (6) and (7) follow from equations (4) and (5).

$\underline{\mathrm{ix}) \Rightarrow \mathrm{xi})}$. The inverse of $\varepsilon$ is given by

$$
\varepsilon^{-1}(g)=\sum_{i} x_{i}\left(\left(\lambda_{i}\right) g\right)
$$

xi $\Rightarrow$ vii $)$. Define $\omega$ by $\omega\left(\lambda \otimes_{S} x\right)=\left(\lambda(\varepsilon(x))\right.$ and put $\sum_{i} x_{i} \otimes_{R} \lambda_{i}=\Delta\left(1_{R}\right)$. ix $\Rightarrow$ xiii). It is clear that $\widetilde{\varepsilon}$ is a morphism in ${ }_{R} \mathcal{M}_{S}$, and from the proof of 
$i x) \Rightarrow x i)$, it follows that $\varepsilon$ is the inverse of $\widetilde{\varepsilon}$. Assume that $(\lambda) g=\left(\lambda^{\prime}\right) g$ for all $g \in{ }_{S} \operatorname{Hom}(\Lambda, \mathrm{S})$. Using equation (6), we find

$$
\lambda=\sum_{i}(\lambda)\left(\varepsilon\left(x_{i}\right)\right) \lambda_{i}=\sum_{i}\left(\lambda^{\prime}\right)\left(\varepsilon\left(x_{i}\right)\right) \lambda_{i}=\lambda^{\prime}
$$

xiii) $\Rightarrow$ ix). Assume that $\widetilde{\varepsilon}$ has an inverse, and call it $\varepsilon$. Then for all $x \in X$, we have

$$
x=\widetilde{\varepsilon}(\varepsilon(x))=\sum_{i} x_{i}\left(\left(\lambda_{i}\right) \varepsilon(x)\right)
$$

and equation (6) follows. For all $g \in{ }_{S} \operatorname{Hom}(\Lambda, S)$, we have

$$
\begin{aligned}
(\lambda) g & =(\lambda)(\varepsilon(\widetilde{\varepsilon}(g))) \\
& =(\lambda)\left(\varepsilon\left(\sum_{i} x_{i}\left(\left(\lambda_{i}\right) g\right)\right)\right) \\
& =\sum_{i}\left((\lambda)\left(\varepsilon\left(x_{i}\right)\right)\right)\left(\lambda_{i}\right) g \\
& =\left((\lambda)\left(\varepsilon\left(x_{i}\right)\right) \lambda_{i}\right) g
\end{aligned}
$$

and equation (7) follows also.

The proof of the implications ii) $\Leftrightarrow$ vii $\Rightarrow \mathrm{x}) \Rightarrow \mathrm{xii}) \Rightarrow$ vii), iii $) \Leftrightarrow$ iv) $\Leftrightarrow$ vi and $\mathrm{x}) \Leftrightarrow \mathrm{xiv}$ ) is similar.

Remarks 2.3. 1) At the beginning of this Section, we have already mentioned that a functor $F$ with a right adjoint between ${ }_{R} \mathcal{M}$ and ${ }_{S} \mathcal{M}$ is isomorphic to $\Lambda \otimes_{R} \bullet$ for some $(S, R)$-bimodule $\Lambda$, and of course we have a similar property for functors beween $\mathcal{M}_{S}$ and $\mathcal{M}_{R}$. If we combine this observation with Theorem 2.2. then we see that there is a one-to-one correspondence between adjoint pairs between ${ }_{R} \mathcal{M}$ and ${ }_{S} \mathcal{M}$, and between $\mathcal{M}_{S}$ and $\mathcal{M}_{R}$.

2) The adjunctions in Theorem 2.2 are equivalences if and only if the unit and counit are natural isomorphisms, and, using Lemma 2.1, we see that this means that the maps $\omega: \Lambda \otimes_{R} X \rightarrow S$ and $\Delta: R \rightarrow X \otimes_{S} \Lambda$ from part vii) of Theorem 2.2 have to be isomorphisms. Using equations (6) and (77), we see that $\left(R, S, X, \Lambda, \Delta^{-1}, \omega\right)$ is a strict Morita contex (recall that the Morita context $\left(R, S, X, \Lambda, \Delta^{-1}, \omega\right)$ is strict if the maps $\Delta^{-1}$ and $\omega$ are isomorphisms). Thus we recover the classical result due to Morita that (additive) equivalences between module categories correspond to strict Morita contexts.

Take $U \in{ }_{R} \mathcal{M}_{R}$ and $V \in{ }_{S} \mathcal{M}_{S}$, and consider the functors

$$
\begin{aligned}
\alpha & =U \otimes_{R} \bullet:{ }_{R} \mathcal{M} \rightarrow{ }_{R} \mathcal{M} ; \beta=V \otimes_{S} \bullet:{ }_{S} \mathcal{M} \rightarrow{ }_{S} \mathcal{M} \\
\alpha^{\prime} & =\bullet \otimes_{R} U: \mathcal{M}_{R} \rightarrow \mathcal{M}_{R} ; \beta^{\prime}=\bullet \otimes_{S} V: \mathcal{M}_{S} \rightarrow \mathcal{M}_{S}
\end{aligned}
$$

Theorem 2.2 allows us to determine when the pair of functors $(F, G)$ is $(\alpha, \beta)$-Frobenius. This is equivalent to one of the 14 equivalent conditions of the Theorem, combined with one of the 14 equivalent conditions of the 
Theorem, but applied to the functors $\left(X \otimes_{S} \bullet, V \otimes_{S} \Lambda \otimes_{R} U \otimes_{R} \bullet\right)$, that is, with $R$ replaced by $S, S$ by $R, \Lambda$ by $X$ and $X$ by $V \otimes_{S} \Lambda \otimes_{R} U$. Thus we obtain 196 equivalent conditions. Moreover, we know from Remark 2.3 2) that autoequivalences of ${ }_{R} \mathcal{M}$ and of ${ }_{S} \mathcal{M}$ correspond to strict Morita contexts. Thus we obtain the following structure Theorem for Frobenius functors of the second kind between categories of modules.

Theorem 2.4. Let $R$ and $S$ be rings. There is one-to-one correspondence between

- Frobenius functors of the second kind between ${ }_{R} \mathcal{M}$ and ${ }_{S} \mathcal{M}$;

- Frobenius functors of the second kind between $\mathcal{M}_{R}$ and $\mathcal{M}_{S}$;

- Fourtuples $(X, \Lambda, \underline{U}, \underline{V})$ where

$$
X \in{ }_{R} \mathcal{M}_{S} ; \Lambda \in{ }_{S} \mathcal{M}_{R}
$$

and

$$
\underline{U}=\left(R, R, U, U^{\prime}, f, f^{\prime}\right) \text { and } \underline{V}=\left(S, S, V, V^{\prime}, g, g^{\prime}\right)
$$

are strict Morita contexts satisfying one of the following equivalent conditions:

i) $X$ is finitely generated projective on the two sides, and we have $(S, R)$ bimodule isomorphisms

$$
\Lambda \cong \operatorname{Hom}_{\mathrm{S}}(\mathrm{X}, \mathrm{S}) \cong \mathrm{V}^{\prime} \otimes_{\mathrm{S} \mathrm{R}} \operatorname{Hom}(\mathrm{X}, \mathrm{R}) \otimes_{\mathrm{R}} \mathrm{U}^{\prime}
$$

ii) $\Lambda$ is finitely generated projective on the two sides, and we have bimodule isomorphisms

$$
X \cong{ }_{S} \operatorname{Hom}(\Lambda, \mathrm{S}) \cong \operatorname{Hom}_{\mathrm{R}}\left(\mathrm{V} \otimes_{\mathrm{S}} \Lambda \otimes_{\mathrm{R}} \mathrm{U}, \mathrm{R}\right)
$$

Remarks 2.5. 1) From Theorem 2.4, we immediately recover the structure Theorem for Frobenius functors of the first kind between module categories (cf. [12, Theorem 2.1], 23, Theorem 3.8]): it suffices to take $V=S$ and $U=R$.

2) If $(X, \Lambda, \underline{U}, \underline{V})$ satisfy the conditions of Theorem 2.4 then we will say that $(X, \Lambda)$ is a $(U, V)$-Frobenius pair of bimodules.

Proposition 2.6. Let $(X, \Lambda)$ be a $(U, V)$-Frobenius pair of bimodules, and consider two other strict Morita contexts $\underline{\widetilde{U}}$ and $\underline{\widetilde{V}}$ on $R$ and $S$.

$(X, \Lambda)$ is a $(\widetilde{U}, V)$-Frobenius pair of bimodules if and only if $\Lambda \otimes_{R} U \cong \Lambda \otimes_{R} \widetilde{U}$ in ${ }_{S} \mathcal{M}_{R}$.

$(X, \Lambda)$ is a $(U, \widetilde{V})$-Frobenius pair of bimodules if and only if $V \otimes_{S} \Lambda \cong \widetilde{V} \otimes_{S} \Lambda$ in ${ }_{S} \mathcal{M}_{R}$.

Proof. Assume that $(X, \Lambda)$ is $(\widetilde{U}, V)$-Frobenius. From condition 1$)$ in Theorem 2.4, it follows that

$$
V \otimes_{S} \Lambda \otimes_{R} U \cong{ }_{R} \operatorname{Hom}(\mathrm{X}, \mathrm{R}) \cong \mathrm{V} \otimes_{\mathrm{S}} \Lambda \otimes_{\mathrm{R}} \widetilde{\mathrm{U}}
$$

in ${ }_{S} \mathcal{M}_{R}$, and

$\Lambda \otimes_{R} U \cong S \otimes_{S} \Lambda \otimes_{R} U \cong V^{\prime} \otimes_{S} V \otimes_{S} \Lambda \otimes_{R} U \cong V^{\prime} \otimes_{S} V \otimes_{S} \Lambda \otimes_{R} \widetilde{U} \cong \Lambda \otimes_{R} \widetilde{U}$ 
in ${ }_{S} \mathcal{M}_{R}$. The converse follows in the same way from condition 1) in Theorem 2.4. The proof of the second statement is similar.

Application to ring extensions. Let $R$ and $S$ be rings, and $i: R \rightarrow S$ a ring homomorphism. We take $\Lambda=S$ considered as an $(S, R)$-bimodule, and $X=S$ considered as an $(R, S)$-bimodule. Then $F=S \otimes_{R} \bullet$ is the induction functor, and $G_{2}$ is the restriction of scalars functor. The conditions of Theorem 2.2 are trivially fulfilled, reflecting the well-known fact that the induction functor is a left adjoint of restriction of scalars.

Theorem 2.7. Let $i: R \rightarrow S$ a ring homomorphism. The induction functor $F=S \otimes_{R} \bullet:{ }_{R} \mathcal{M} \rightarrow{ }_{S} \mathcal{M}$ and the restriction of scalars functor ${ }_{S} \mathcal{M} \rightarrow{ }_{R} \mathcal{M}$ form a Frobenius pair of the second kind if and only if there exist Morita contexts $\underline{U}$ and $\underline{V}$ (with notation as in Theorem 2.4) such that one of the following equivalent conditions hold:

i)

ii)

$$
V \otimes_{R} U \otimes_{R} \bullet \cong{ }_{R} \operatorname{Hom}(\mathrm{S}, \bullet)
$$

$$
\text { - } \otimes_{R} S \cong \operatorname{Hom}_{\mathrm{R}}\left(\mathrm{V} \otimes_{\mathrm{R}} \mathrm{U}, \bullet\right)
$$

iii) $S$ is finitely generated and projective as a left $R$-module and

$$
V \otimes_{R} U \cong{ }_{R} \operatorname{Hom}(\mathrm{S}, \mathrm{R}) \text { in }{ }_{\mathrm{S}} \mathcal{M}_{\mathrm{R}}
$$

iv) $S$ is finitely generated and projective as a right $R$-module and

$$
S \cong \operatorname{Hom}_{\mathrm{R}}\left(\mathrm{V} \otimes_{\mathrm{R}} \mathrm{U}, \mathrm{R}\right) \text { in }{ }_{\mathrm{R}} \mathcal{M}_{\mathrm{S}}
$$

v) there exist $z=\sum_{i} v_{i} \otimes_{R} u_{i} \otimes_{R} s_{i} \in C_{S}\left(V \otimes_{R} U \otimes_{R} S\right)$ and $\omega: V \otimes_{R} U \rightarrow R$ in ${ }_{R} \mathcal{M}_{R}$ such that

$$
\begin{aligned}
& \sum_{i} \omega\left(v_{i} \otimes_{R} u_{i}\right) s_{i}=1 \\
& \sum_{i} v_{i} \otimes_{R} u_{i} \omega\left(s_{i} v \otimes_{R} u\right)=v \otimes_{R} u
\end{aligned}
$$

for all $u \in U$ and $v \in V$.

vi) as $v$ ), with $z \in V \otimes_{R} U \otimes_{R} S$ and (10) replaced by

$$
\sum_{i} \omega\left(s v_{i} \otimes_{R} u_{i}\right) s_{i}=s
$$

for all $s \in S$.

We will call the extension $S / R(U, V)$-Frobenius.

Remarks 2.8. 1) Taking $U=R$ and $V=S$ in Theorem 2.7 we find necessary and sufficient conditions for the induction and restriction of scalars functors form to be a Frobenius pair. In particular, this shows that $S$ is a Frobenius extension of $R$ (in the sense of e.g. [16, Definition 2.1]) if and only if the induction functor is Frobenius.

2) From Proposition 2.6. we immediately obtain the following: assume that $S / R$ is a $(U, V)$-Frobenius extension. Then $S / R$ is $(\widetilde{U}, V)$-Frobenius if and 
only if $S \otimes_{R} U \cong S \otimes_{R} \widetilde{U}$ in ${ }_{S} \mathcal{M}_{R}$.

$S / R$ is $(U, \widetilde{V})$-Frobenius if and only if $V \cong \widetilde{V}$ in ${ }_{S} \mathcal{M}_{R}$.

Example 2.9. Let $\mu$ and $\varphi$ be ring automorphisms of $R$. For every $R$ bimodule $M$, we introduce a new $R$-bimodule ${ }_{\mu} M_{\varphi}$ as follows: ${ }_{\mu} M_{\varphi}=M$ as an abelian group, but with left and right $R$-action

$$
r \cdot m \cdot r^{\prime}=\mu(r) m \varphi\left(r^{\prime}\right)
$$

If $\mu$ and/or $\varphi$ is the identity, then we omit the index, for example ${ }_{\mu} M_{I_{R}}=$ ${ }_{\mu} M$. It is easy to show that ${ }_{\varphi \mu} M \cong{ }_{\varphi}\left({ }_{\mu} M\right)$, and that $M_{\varphi} \otimes_{R} N \cong M \otimes_{R \varphi^{-1}} N$. Furthermore, we have a strict Morita context $\left(R, R,{ }_{\mu} R, R_{\mu}, f, g\right)$.

Now let $\mu: R \rightarrow R$ and $\nu: S \rightarrow S$ be ring automorphisms. In Theorem 2.7 we take $U={ }_{\mu} R$ and $V={ }_{\nu} S$. We then have

$$
V \otimes_{R} U \cong{ }_{\nu} S_{\mu^{-1}} \text { and } \operatorname{Hom}_{\mathrm{R}}\left(\mathrm{V} \otimes_{\mathrm{R}} \mathrm{U}, \mathrm{M}\right) \cong \operatorname{Hom}_{\mathrm{R}}\left({ }_{\nu} \mathrm{S}, \mathrm{R}_{\mu}\right)
$$

An $\left(R_{\mu}, S_{\nu}\right)$-Frobenius extension will also be called a $(\mu, \nu)$-Frobenius extension. From Theorem 2.7, we see that this is equivalent to each of the following equivalent conditions.

i)

ii)

$$
{ }_{\nu} S \otimes_{R \mu}(\bullet) \cong{ }_{R} \operatorname{Hom}(\mathrm{S}, \bullet)
$$

$$
\text { - } \otimes_{R} S \cong \operatorname{Hom}_{\mathrm{R}}\left({ }_{\nu} \mathrm{S},(\bullet)_{\mu}\right)
$$

iii) $S$ is finitely generated and projective as a left $R$-module, and ${ }_{\nu} S_{\mu^{-1}} \cong$ ${ }_{R} \operatorname{Hom}(\mathrm{S}, \mathrm{R})$ in ${ }_{S} \mathcal{M}_{R}$.

iv) $S$ is finitely generated and projective as a right $R$-module, and $S \cong$ $\operatorname{Hom}_{\mathrm{R}}\left({ }_{\nu} \mathrm{S}, \mathrm{R}_{\mu}\right)$ in ${ }_{R} \mathcal{M}_{S}$.

v) There exist $x_{i}, s_{i} \in S$ and $\omega:{ }_{\nu} S_{\mu^{-1}} \rightarrow R$ in ${ }_{R} \mathcal{M}_{R}$ such that

$$
s=\sum_{i} \omega\left(\nu(s) x_{i}\right) s_{i}=\sum_{i} x_{i} \mu^{-1}\left(\omega\left(\nu\left(s_{i}\right) s\right)\right)
$$

for all $s \in S$.

In this case $\sum_{i} x_{i} \otimes s_{i} \in C_{S}\left({ }_{\nu} S_{\mu^{-1}} \otimes_{R} S\right)$.

If $\nu=I_{S}$, then we recover the definition of Frobenius extension of the second kind from [16, Ch. 7] and [25].

Let $S / R$ be a $(\mu, S)$-Frobenius extension, and consider another automorphism $\widetilde{\mu}$ of $R$. Then $S / R$ is $(\widetilde{\mu}, S)$-Frobenius if and only if

$$
S \otimes_{R \mu} R \cong S \otimes_{R \widetilde{\mu}} R \text { or } S_{\mu^{-1}} \cong S_{\widetilde{\mu}^{-1}} \text { in }{ }_{S} \mathcal{M}_{R}
$$

Let $\alpha: S_{\mu^{-1}} \rightarrow S_{\widetilde{\mu}^{-1}}$ be the connecting $(S, R)$-isomorphism, and let $\alpha\left(1_{S}\right)=$ $u, \alpha^{-1}\left(1_{S}\right)=v \in S$. Then

$$
1_{S}=\alpha\left(v 1_{S}\right)=v \alpha\left(1_{S}\right)=v u \text { and } 1_{S}=\alpha^{-1}\left(u 1_{S}\right)=u \alpha^{-1}(1)=u v
$$

so $v=u^{-1}$. Furthermore

$$
\begin{aligned}
\mu^{-1}(r) u & =\mu^{-1}(r) \alpha\left(1_{S}\right)=\alpha\left(\mu^{-1}(r) 1_{S}\right) \\
& =\alpha\left(1_{S} \mu^{-1}(r)\right)=\alpha\left(1_{S}\right) \widetilde{\mu}^{-1}(r)=u \widetilde{\mu}^{-1}(r)
\end{aligned}
$$


and

$$
\mu^{-1}(r)=u \widetilde{\mu}^{-1}(r) u^{-1} \text { and } \mu(r)=u \widetilde{\mu}(r) u^{-1}
$$

so $\mu$ and $\widetilde{\mu}$ are equal up to an inner automorphism. Compare this to 16 , Prop. 7.3].

\section{Applichtion to Hopf algebras}

Let $k$ be a commutative ring, $H$ a Hopf algebra, and $I$ a projective $k$-module of rank one. We will apply the results of the Section 2 in the situation where $R=k, S=H, U=I$ and $V=H$. If the induction functor and the restriction of scalars functor form a $(U, H)$-Frobenius pair, we say that $H / k$ is $I$-Frobenius. Using Theorem 2.7. we find the following characterization. $\otimes$ will be a shorter notation for $\otimes_{k}$.

Theorem 3.1. Let $H$ be a Hopf algebra over a commutative ring $k$, and $I$ a projective rank one module. Then the following assertions are equivalent:

i) $H / k$ is I-Frobenius;

ii) $H$ is finitely generated and projective as a $k$-module, and

$$
H \otimes I \cong H^{*} \text { in }{ }_{H} \mathcal{M}
$$

iii) $H$ is finitely generated and projective as a $k$-module, and

$$
H \cong(H \otimes I)^{*} \text { in } \mathcal{M}_{H}
$$

iv) There exist $z=e^{1} \otimes x^{1} \otimes e^{2} \in C_{H}(H \otimes I \otimes H)$ and $\omega \in(H \otimes I)^{*}$ such that

$$
\begin{aligned}
& \omega\left(e^{1} \otimes x^{1}\right) e^{2}=1 \\
& e^{1} \otimes x^{1} \omega\left(e^{2} \otimes x\right)=1_{H} \otimes x
\end{aligned}
$$

for all $x \in I$.

Note that $e^{1} \otimes x^{1} \otimes e^{2}$ is a formal notation for an element $\sum_{i} e_{i} \otimes x_{i} \otimes e_{i}^{\prime} \in$ $H \otimes I \otimes H$.

We will give more equivalent conditions for $H / k$ to be $I$-Frobenius. First recall the notion of (left and right) integral in $H$ and on $H$ :

$$
\begin{aligned}
\int_{H}^{l} & =\{t \in H \mid h t=\varepsilon(h) t, \text { for all } h \in H\} \\
\int_{H}^{r} & =\{t \in H \mid t h=\varepsilon(h) t, \text { for all } h \in H\} \\
\int_{H^{*}}^{l} & =\left\{\varphi \in H^{*} \mid h^{*} * \varphi=\left\langle h^{*}, 1\right\rangle \varphi, \text { for all } h^{*} \in H^{*}\right\} \\
\int_{H^{*}}^{r} & =\left\{\varphi \in H^{*} \mid \varphi * h^{*}=\left\langle h^{*}, 1\right\rangle \varphi, \text { for all } h^{*} \in H^{*}\right\}
\end{aligned}
$$

Recall that the multiplication on $H^{*}$ is given by the convolution:

$$
\left\langle h^{*} * k^{*}, h\right\rangle=\left\langle h^{*}, h_{(1)}\right\rangle\left\langle k^{*}, h_{(2)}\right\rangle
$$


The following result follows from the Fundamental Theorem for Hopf modules. The proof may be found in [29] if $k$ is a field, and it can be adapted easily to the situation where $k$ is a commutative ring.

Theorem 3.2. For a finitely generated projective Hopf algebra $H$, we have an isomorphism

$$
\alpha: \int_{H^{*}}^{l} \otimes H \rightarrow H^{*},\langle\alpha(\varphi \otimes h), k\rangle=\langle\varphi, k S(h)\rangle
$$

$\alpha$ is left $H^{*}$-linear and right $H$-linear; the left $H^{*}$-action on $\int_{H^{*}}^{l} \otimes H$, and the right $H$-action on $H^{*}$ are given by

$$
k^{*} \cdot(t \otimes h)=\left\langle k^{*}, h_{(2)}\right\rangle t \otimes h_{(1)} \text { and }\left\langle h^{*} \measuredangle h, k\right\rangle=\left\langle h^{*}, k S(h)\right\rangle
$$

Consequently $\int_{H^{*}}^{l}$ is finitely generated projective of rank one.

Proposition 3.3. Let $H$ be a finitely generated projective Hopf algebra, and $I$ a projective rank one $k$-module. We then have maps

$$
\begin{gathered}
p: \quad C_{H}(H \otimes I \otimes H) \rightarrow \int_{H}^{l} \otimes I, p\left(e^{1} \otimes x^{1} \otimes e^{2}\right)=\varepsilon\left(e^{2}\right) e^{1} \otimes x^{1} \\
p^{\prime}: \quad C_{H}(H \otimes I \otimes H) \rightarrow I \otimes \int_{H}^{r}, p^{\prime}\left(e^{1} \otimes x^{1} \otimes e^{2}\right)=\varepsilon\left(e^{1}\right) x^{1} \otimes e^{2} \\
\quad i: \quad \int_{H}^{l} \otimes I \rightarrow C_{H}(H \otimes I \otimes H), i(t \otimes x)=t_{(1)} \otimes x \otimes S\left(t_{(2)}\right) \\
i^{\prime}: \quad I \otimes \int_{H}^{r} \rightarrow C_{H}(H \otimes I \otimes H), i^{\prime}(x \otimes t)=S\left(t_{(1)}\right) \otimes x \otimes t_{(2)}
\end{gathered}
$$

$i$ and $i^{\prime}$ are sections for $p$ and $p^{\prime}$.

Proof. The multiplication on $H$ induces a right $H^{*}$-coaction on $H$ :

$$
\rho^{r}(h)=h_{[0]} \otimes h_{[1]} \in H \otimes H^{*} \Longleftrightarrow k h=\left\langle h_{[1]}, k\right\rangle h_{[0]}, \text { for all } k \in H
$$

Then $\int_{H}^{l}$ is the kernel of $\rho^{r}-I_{H} \otimes \eta_{H^{*}} . I$ is projective, and flat, so $\int_{H}^{l} \otimes I$ is the kernel of the map $\rho^{r} \otimes I_{I}-I_{H} \otimes \eta_{H^{*}} \otimes I_{I}$, i.e.

$$
\int_{H}^{l} \otimes I=\left\{\sum_{i} u_{i} \otimes x_{i} \in H \otimes I \mid \sum_{i} h u_{i} \otimes x_{i}=\sum_{i} \varepsilon(h) u_{i} \otimes x_{i} \text { for all } h \in H\right\}
$$

We use this to prove that $p\left(e^{1} \otimes x^{1} \otimes x^{2}\right) \in \int_{H}^{l} \otimes I$ :

$$
h \varepsilon\left(e^{2}\right) e^{1} \otimes x^{1}=\varepsilon\left(e^{2} h\right) e^{1} \otimes x^{1}=\varepsilon(h) \varepsilon\left(e^{2}\right) e^{1} \otimes x^{1}
$$

Now take $t \otimes x \in \int_{H}^{l} \otimes I$. Then $i(t \otimes x) \in C_{H}(H \otimes I \otimes H)$ since

$$
\begin{aligned}
h t_{(1)} \otimes & \otimes S\left(t_{(2)}\right)=h_{(1)} t_{(1)} \otimes x \otimes S\left(t_{(2)}\right) S\left(h_{(2)}\right) h_{(3)} \\
& =\left(h_{(1)} t\right)_{(1)} \otimes x \otimes S\left(\left(h_{(1)} t\right)_{(2)}\right) h_{(2)} \\
& =\left(\varepsilon\left(h_{(1)}\right) t\right)_{(1)} \otimes x \otimes S\left(\left(\varepsilon\left(h_{(1)}\right) t\right)_{(2)}\right) h_{(2)} \\
& =t_{(1)} \otimes x \otimes S\left(t_{(2)}\right) h
\end{aligned}
$$


We leave it to the reader to prove that $i$ is a section of $p$. The proof for $p^{\prime}$ and $i^{\prime}$ is similar.

We can now state and prove the main result of this Section. In the situation where $I=k$ is free of rank one, we recover a result of Pareigis [26]. It follows from Theorem 3.4 that a finitely generated projective Hopf algebra is a Frobenius extension of $k$ of the second kind, a result that goes back to Pareigis [27, and that has been revived recently by Kadison and Stolin, see [17. Prop. 3.5].

Theorem 3.4. Let $H$ be Hopf algebra over a commutative ring $k$ and $I$ a projective $k$-module of rank one. Then the following assertions are equivalent.

i) $H / k$ is I-Frobenius;

ii) $H$ is finitely generated and projective and $H^{*} / k$ is $I^{*}$-Frobenius;

iii) $H$ is finitely generated and projective and $\int_{H}^{l} \cong I^{*}$;

iv) $H$ is finitely generated and projective and $\int_{H}^{r} \cong I^{*}$;

v) $H$ is finitely generated and projective and $\int_{H^{*}}^{l} \cong I$;

vi) $H$ is finitely generated and projective and $\int_{H^{*}}^{r} \cong I$.

Proof. i) $\Rightarrow$ iii). Take $z=\sum e^{1} \otimes x^{1} \otimes e^{2} \in C_{H}(H \otimes I \otimes H)$ and $\omega \in(H \otimes I)^{*}$ as in Theorem 3.1] and put

$$
t=p(z)=\varepsilon\left(e^{2}\right) e^{1} \otimes x^{1} \in \int_{H}^{l} \otimes I
$$

For an arbitrary $u=\sum_{i} u_{i} \otimes x_{i} \in \int_{H}^{l} \otimes I$, we have

$$
\begin{aligned}
\omega(u) t & =\omega\left(\sum_{i} u_{i} \otimes x_{i}\right) \varepsilon\left(e^{2}\right) e^{1} \otimes x^{1} \\
& =\omega\left(\sum_{i} \varepsilon\left(e^{2}\right) u_{i} \otimes x_{i}\right) e^{1} \otimes x^{1} \\
& =\omega\left(\sum_{i} e^{2} u_{i} \otimes x_{i}\right) e^{1} \otimes x^{1} \\
& =\omega\left(\sum_{i} e^{2} \otimes x_{i}\right) u_{i} e^{1} \otimes x^{1} \\
& =u_{i} e^{1} \otimes x^{1} \omega\left(\sum_{i} e^{2} \otimes x_{i}\right) \\
& =\sum_{i} u_{i} \otimes x_{i}=u
\end{aligned}
$$

This means that the map $k \rightarrow \int_{H}^{l} \otimes I$ sending $y \in k$ to $y t$ is surjective. This map is also injective: if

$$
y t=y \varepsilon\left(e^{2}\right) e^{1} \otimes x^{1}=0
$$


then

$$
0=\omega\left(y \varepsilon\left(e^{2}\right) e^{1} \otimes x^{1}\right)=y \omega\left(e^{1} \otimes x^{1}\right) \varepsilon\left(e^{2}\right)=y \varepsilon(1)=y
$$

where we used (12). We have shown that $\int_{H}^{l} \otimes I \cong k$, which is equivalent to $\int_{H}^{l} \cong I^{*}$.

v) $\Rightarrow$ ii). Consider the isomorphism

$$
\alpha: \int_{H^{*}}^{l} \otimes H \rightarrow H^{*}
$$

from Theorem 3.2. This induces an isomorphism

$$
\beta: H \rightarrow H^{*} \otimes I^{*}
$$

$\beta$ is left $H^{*}$-linear, so it follows from ii) of Theorem 3.1 (with $H$ replaced by $H^{*}$ ) that $H^{*} / k$ is $I^{*}$-Frobenius.

v) $\Rightarrow$ i). Remark that the antipode of a finitely generated projective Hopf

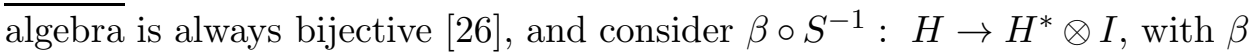
as above. Then $\beta \circ S^{-1}$ is bijective, and we are done if we can show that it is also left $H$-linear, if the left $H$-action on $H^{*} \otimes I$ is given by

$$
h \cdot\left(k^{*} \otimes x\right)=h \cdot k^{*} \otimes x, \text { with }\left\langle h \cdot k^{*}, k\right\rangle=\left\langle k^{*}, k h\right\rangle
$$

Using the right $H$-linearity in Theorem 3.2 , we compute

$$
\left(\beta \circ S^{-1}\right)(h k)=\beta\left(S^{-1}(k) S^{-1}(h)\right)=\beta\left(S^{-1}(k)\right) S\left(S^{-1}(h)\right)=\beta\left(S^{-1}(k)\right) h
$$

as needed.

ii) $\Rightarrow$ v) follows after we apply i) $\Rightarrow$ iii) with $H$ replaced by $H^{*}$.

iii) $\Rightarrow$ ii) and iii) $\Rightarrow$ i) follow after we apply v) $\Rightarrow$ i) and v) $\Rightarrow$ ii) with $H^{*}$ replaced by $H$.

iii) $\Leftrightarrow$ iv). Observe that $S: \int_{H}^{l} \rightarrow \int_{H}^{r}$ is a bijection; in the same way v) $\Leftrightarrow$ vi) follows.

It is well-known that, for a finitely generated projective Hopf algebra, the integral space is a projective module of rank one. From Theorem 3.4 it follows that the integral spaces of $H$ and $H^{*}$ are isomorphic, i.e. they represent the same element in the Picard group of $k$. Of course this is trivial in the case where $k$ is a field, because then every rank one projective module is free, but, as far as we could figure out, it is a new result in the case where $k$ is a commutative ring.

Corollary 3.5. Let $H$ be a finitely generated projective Hopf algebra over a commutative ring $k$. Then we have isomorphisms of $k$-modules

$$
\int_{H^{*}}^{l} \cong \int_{H^{*}}^{r} \cong\left(\int_{H}^{l}\right)^{*} \cong\left(\int_{H}^{r}\right)^{*}
$$




\section{Comodules over coalgebras}

Let $C$ and $D$ be coalgebras over a field $k$, and consider two bicomodules $\Lambda \in{ }^{D} \mathcal{M}^{C}$ and $X \in{ }^{C} \mathcal{M}^{D}$. The functor

$$
G: \mathcal{M}^{D} \rightarrow \mathcal{M}^{C} ; G(N)=N \square_{D} \Lambda
$$

has a left adjoint $F$ if and only if $\Lambda$ is quasifinite as a right $C$-comodule, this means that $\operatorname{Hom}^{\mathrm{C}}(\mathrm{V}, \Lambda)$ is finite dimensional for every finite dimensional right $C$-comodule $V$. If $\Lambda$ is finitely cogenerated as a $C$-comodule, then $\Lambda$ is quasifinite. If $C$ is finite dimensional, then every $C$-comodule is quasifinite. The functor $F$ can be given an explicit description, which is complicated, and we will not need it. Details can be found in [30, Sec. 1]. The functor $F$ is called the co-Hom functor, and we write

$$
F(M)=\mathrm{h}_{\mathrm{C}}(\Lambda, \mathrm{M})
$$

$\mathrm{h}_{\mathrm{C}}$ is covariant in $M$ and contravariant in $\Lambda$. Essentially every adjoint pair of functors is of this type: assume that $(F, G)$ is an adjoint pair of $k$-linear functors between $\mathcal{M}^{C}$ and $\mathcal{M}^{D}$. Then $G$ is left exact and preserves direct sums, and by [30, Prop. 2.1], $G \cong \bullet \square_{D} \Lambda$ for some bicomodule $\Lambda \in{ }^{D} \mathcal{M}^{C}$. It follows that $\Lambda$ is then quasifinite as a right $C$-comodule, and $F \cong \mathrm{h}_{\mathrm{C}}(\Lambda, \bullet)$.

Lemma 4.1. Consider two $(D, C)$-bicomodules $\Lambda$ and $\Lambda^{\prime}$, and the functors $G=\bullet \square_{D} \Lambda$ and $G^{\prime}=\bullet \square_{D} \Lambda^{\prime}$. then

$$
\underline{\underline{\operatorname{Nat}}}\left(G, G^{\prime}\right) \cong{ }^{D} \operatorname{Hom}^{\mathrm{C}}\left(\Lambda, \Lambda^{\prime}\right)
$$

Proof. Essentially this is [30, 2.2]. We include more detail for the sake of completeness. First a natural transformation $\alpha: G \rightarrow G^{\prime}$. We will show that $\alpha$ is determined completely by $\alpha_{D}$ and that $\alpha_{D}$ is bicolinear.

For any $k$-module $M, M \otimes D \in \mathcal{M}^{D}$, with coaction induced by the comultiplication on $D$. For any $m \in M, f_{m}: D \rightarrow M \otimes D, f_{m}(d)=m \otimes d$, is right $D$-colinear, and the naturality of $\alpha$ gives a commutative diagram

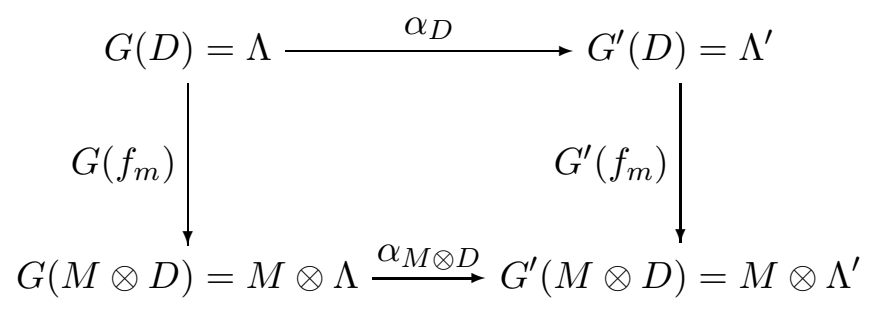

Clearly $G\left(f_{m}\right)(\lambda)=m \otimes \lambda$, and it follows that $\alpha_{M \otimes D}=I_{M} \otimes \alpha_{D}$. Now consider an arbitrary $M \in \mathcal{M}^{D}$. The coaction map $\rho_{M}^{r}: M \rightarrow M \otimes D$ 
is in $\mathcal{M}^{D}$, so we find a commutative diagram

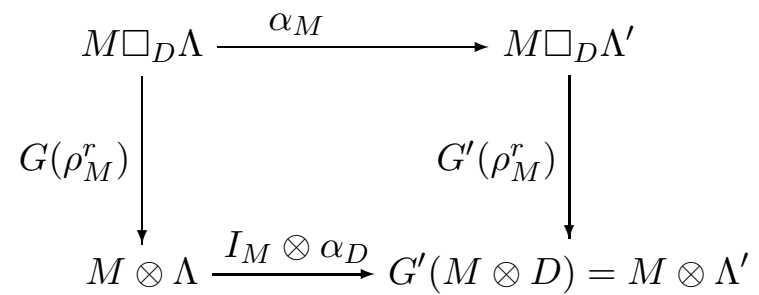

$G\left(\rho_{M}^{r}\right)$ is the inclusion map, and we find that $\alpha_{M}=I_{M} \square_{D} \alpha_{D}$, proving that $\alpha$ is completely determined by $\alpha_{D} \cdot \alpha_{D}$ is right $C$-colinear, and the naturality of $\alpha$ implies that $\alpha_{D}$ is also left $D$-colinear. Indeed, the comultiplication $\Delta_{D}: D \rightarrow D \otimes D$ is a map in $\mathcal{M}^{D}$, and $G\left(\Delta_{D}\right)=\rho_{\Lambda}^{l}, G^{\prime}\left(\Delta_{D}\right)=\rho_{\Lambda^{\prime}}^{l}$, the left $D$-coaction on $\Lambda$ and $\Lambda^{\prime}$. The naturality of $\alpha$ implies that we have a commutative diagram

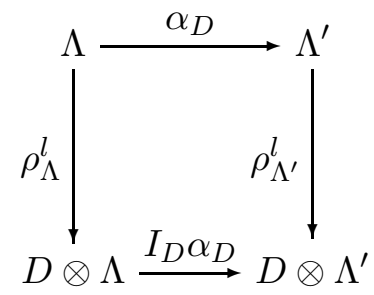

and it follows that $\alpha_{D}$ is left $D$-colinear.

Given $\widetilde{\alpha} \in{ }^{D} \operatorname{Hom}^{\mathrm{C}}\left(\Lambda, \Lambda^{\prime}\right)$, we define $\alpha: G \rightarrow G^{\prime}$ by $\alpha_{M}=I_{M} \square_{D} \widetilde{\alpha}$. It is then clear that $\alpha$ is natural.

Let $\Lambda \in{ }^{D} \mathcal{M}^{C}$ be quasifinite as a right $C$-comodule. By [30, 1.9], $\mathrm{h}_{\mathrm{C}}(\Lambda, \mathrm{C}) \in$ ${ }^{\mathrm{C}} \mathcal{M}^{\mathrm{D}}$, and we can consider the functor

$$
F_{1}: \mathcal{M}^{C} \rightarrow \mathcal{M}^{D}, F_{1}(M)=M \square_{C} \mathrm{~h}_{\mathrm{C}}(\Lambda, \mathrm{C})
$$

For all $M \in \mathcal{M}^{C}$, we have isomorphisms

$\operatorname{Hom}^{\mathrm{D}}\left(\mathrm{F}(\mathrm{M}), \mathrm{F}_{1}(\mathrm{M})\right) \cong \operatorname{Hom}^{\mathrm{C}}\left(\mathrm{M}, \mathrm{GF}_{1}(\mathrm{M})\right)=\operatorname{Hom}^{\mathrm{C}}\left(\mathrm{M}, \mathrm{M}_{\mathrm{Ch}} \mathrm{h}_{\mathrm{C}}(\Lambda, \mathrm{C}) \square_{\mathrm{D}} \Lambda\right)$

hence

and, using Lemma 4.1

$$
\underline{\underline{\mathrm{Nat}}}\left(F, F_{1}\right) \cong \underline{\underline{\operatorname{Nat}}}\left(1_{\mathcal{M}^{C}}, \bullet \square_{C} \mathrm{~h}_{\mathrm{C}}(\Lambda, \mathrm{C}) \square_{\mathrm{D}} \Lambda\right)
$$

$$
\underline{\underline{\operatorname{Nat}}}\left(F, F_{1}\right) \cong{ }^{C} \operatorname{Hom}^{\mathrm{C}}\left(\mathrm{C}, \mathrm{h}_{\mathrm{C}}(\Lambda, \mathrm{C}) \square_{\mathrm{D}} \Lambda\right)
$$

Applying the unit of the adjunction $(F, G)$ to $C \in \mathcal{M}^{C}$, we find a bicolinear map

$$
\eta_{C}: C \rightarrow G F(C)=\mathrm{h}_{\mathrm{C}}(\Lambda, \mathrm{C}) \square_{\mathrm{D}} \Lambda
$$

and associated to this is a natural transformation

$$
\gamma: F \rightarrow F_{1}
$$

which can be considered as the dual version of the natural transformation $\gamma: G_{1} \rightarrow G$ introduced in Section 2] the difference is that the $\gamma$ from Section 2 could be described explicitely, while here we have to make use of 
the adjunction properties. It follows from [30, Prop. 1.14] that $\gamma$ is a natural isomorphism if $\Lambda$ is injective as a right $C$-comodule.

Now let $\Lambda \in{ }^{D} \mathcal{M}^{C}$ and $X \in{ }^{C} \mathcal{M}^{D}$, and consider the functors

$$
\begin{aligned}
& G=\bullet \square_{D} \Lambda: \mathcal{M}^{D} \rightarrow \mathcal{M}^{C} ; F_{2}=\bullet \square_{C} X: \mathcal{M}^{C} \rightarrow \mathcal{M}^{D} \\
& G^{\prime}=X \square_{D} \bullet:{ }^{D} \mathcal{M} \rightarrow{ }^{C} \mathcal{M} ; F_{2}^{\prime}=\Lambda \square_{C} \bullet:{ }^{C} \mathcal{M} \rightarrow{ }^{D} \mathcal{M}
\end{aligned}
$$

If $\Lambda$ is quasifinite as a right $C$-comodule, then we also consider

$$
F=\mathrm{h}_{\mathrm{C}}(\Lambda, \bullet), \mathrm{F}_{1}=\bullet \square_{\mathrm{C}} \mathrm{h}_{\mathrm{C}}(\Lambda, \mathrm{C}): \mathcal{M}^{\mathrm{C}} \rightarrow \mathcal{M}^{\mathrm{D}}
$$

and if $X$ is quasifinite as a left $C$-comodule, then we consider

$$
F^{\prime}={ }_{C} \mathrm{~h}(\mathrm{X}, \bullet), \mathrm{F}_{1}^{\prime}={ }_{\mathrm{C}} \mathrm{h}(\mathrm{X}, \mathrm{C}) \square_{\mathrm{D}} \bullet:{ }^{\mathrm{C}} \mathcal{M} \rightarrow{ }^{\mathrm{D}} \mathcal{M}
$$

and we have a natural transformation $\gamma^{\prime}: F^{\prime} \rightarrow F_{1}^{\prime}$ that is a natural isomorphism if $X$ is injective as a left $C$-comodule. The following result is then the coalgebra version of Morita's Theorem 2.2

Theorem 4.2. Let $C$ and $D$ be coalgebras over a field $k, \Lambda \in{ }^{D} \mathcal{M}^{C}$ and $X \in{ }^{C} \mathcal{M}^{D}$. Using the notation introduced above, we consider the following properties.

i) $\left(F_{2}=\bullet \square_{C} X, G=\bullet \square_{D} \Lambda\right)$ is an adjoint pair of functors;

ii) ( $\left.F_{2}^{\prime}=\Lambda \square_{C} \bullet, G^{\prime}=X \square_{D} \bullet\right)$ is an adjoint pair of functors;

iii) $\Lambda$ is quasifinite as a right $C$-comodule, and the functors $F_{2}$ and $F$ are naturally isomorphic;

iv) $X$ is quasifinite as a left $C$-comodule, and the functors $F_{2}^{\prime}$ and $F^{\prime}$ are naturally isomorphic;

v) there exist bicolinear maps

$$
\psi: C \rightarrow X \square_{D} \Lambda \text { and } \omega: \Lambda \square_{C} X \rightarrow D
$$

respectively in ${ }^{C} \mathcal{M}^{C}$ and ${ }^{D} \mathcal{M}^{D}$ such that

$$
\begin{aligned}
\left(\omega \square_{D} I_{\Lambda}\right) \circ\left(I_{\Lambda} \square_{C} \psi\right) & =I_{\Lambda} \\
\left(I_{X} \square_{D} \omega\right) \circ\left(\psi \square_{C} I_{X}\right) & =I_{X}
\end{aligned}
$$

vi) $\Lambda$ is quasifinite and injective as a right $C$-comodule and $X \cong \mathrm{h}_{\mathrm{C}}(\Lambda, \mathrm{C})$ in ${ }^{C} \mathcal{M}^{D}$

vii) $X$ is quasifinite and injective as a left $C$-comodule and $\Lambda \cong{ }_{C} \mathrm{~h}(\Lambda, \mathrm{C})$ in ${ }^{D} \mathcal{M}^{C}$.

The conditions i) to $v$ ) are equivalent. vi) and vii) imply $i-v)$. i-v) $\Rightarrow$ vi) holds if $X$ is quasifinite as a right $D$-comodule. $\mathrm{i}-\mathrm{v}) \Rightarrow$ vii) holds if $\Lambda$ is quasifinite as a left D-comodule.

Proof. i) $\Leftrightarrow \mathrm{v}$ ) is a dual version of the proof of i) $\Leftrightarrow$ vii) in Theorem 2.2

i) $\Leftrightarrow$ iii). If $G$ has a left adjoint, then $\Lambda \in \mathcal{M}^{C}$ is quasifinite; then we use the uniqueness of the adjoint.

vi) $\Rightarrow$ iii). If $X \cong \mathrm{h}_{\mathrm{C}}(\Lambda, \mathrm{C})$, then $F_{2} \cong F_{1}$. If $\Lambda \in \mathcal{M}^{C}$ is quasifinite and injective, then $F_{1} \cong F$.

iii) $\Rightarrow$ vi). Assume that $X$ is quasifinite as a right $D$-comodule. Then $F_{2}$ 
has a left adjoint, namely $\mathrm{h}_{\mathrm{D}}(\mathrm{X}, \bullet)$, and a right adjoint, namely $G$. Thus $F_{2}$ is exact, and $G$ preserves injective objects, and it follows that $G(D)=\Lambda$ is an injective object of $\mathcal{M}^{C}$.

The proof of the other implications is similar (observe that $\mathrm{v}$ ) is left-right symmetric).

Using Theorem 4.2, we can characterize all ( $k$-linear) Frobenius pairs of the second kind between categories of comodules. From [30, we know that category equivalences between $\mathcal{M}^{C}$ and $\mathcal{M}^{D}$ correspond to strict MoritaTakeuchi contexts. A strict Morita-Takeuchi context between $C$ and $D$ is a sixtuple $\left(C, D, \Lambda, X, \psi, \omega^{-1}\right)$ as in part 5) of Theorem 4.2. with the additional condition that $\psi$ and $\omega$ are bijective. Now let $\underline{U}=\left(C, C, U, U^{\prime}, f, f^{\prime}\right)$ and $\underline{V}=\left(D, D, V, V^{\prime}, g, g^{\prime}\right)$ be strict Morita-Takeuchi contexts, and consider the corresponding autoequivalences $\alpha=\bullet \square_{C} U$ and $\beta=\bullet \square_{D} V$ of $\mathcal{M}^{C}$ and $\mathcal{M}^{D}$. Using Theorem 4.2 we can characterize when $\left(G=\bullet \square_{D} \Lambda, \beta \circ F_{2} \circ \alpha\right)=$ - $\left.\square_{C} U \square_{C} X \square_{D} V\right)$ is an adjoint pair. Combining this with Theorem4.2 in its original form, we find necessary and sufficient conditions for $\left(F_{2}, G\right)$ being $(\alpha, \beta)$-Frobenius. We obtain the following result:

Theorem 4.3. Let $C$ and $D$ be coalgebras over a field $k$. Then we have a one-to-one correspondence between

- $k$-linear Frobenius functors of the second kind between $\mathcal{M}^{C}$ and $\mathcal{M}^{D}$;

- $k$-linear Frobenius functors of the second kind between ${ }^{C} \mathcal{M}$ and ${ }^{D} \mathcal{M}$;

- fourtuples $(X, \Lambda, \underline{U}, \underline{V})$ where $\Lambda \in{ }^{D} \mathcal{M}^{C}, X \in{ }^{C} \mathcal{M}^{D}$, and $\underline{U}=$ $\left(C, C, U, U^{\prime}, f, f^{\prime}\right)$ and $\underline{V}=\left(D, D, V, V^{\prime}, g, g^{\prime}\right)$ strict Morita-Takeuchi contexts, such that one of the following equivalent conditions hold:

i) $\Lambda$ and $X$ are quasifinite and injective, respectively as a right $C$ comodule and a right $D$-comodule, and we have bicolinear isomorphisms

$$
X \cong \mathrm{h}_{\mathrm{C}}(\Lambda, \mathrm{C}) \text { and } \Lambda \cong \mathrm{h}_{\mathrm{D}}\left(\mathrm{U} \square_{\mathrm{C}} \mathrm{X} \square_{\mathrm{D}} \mathrm{V}, \mathrm{D}\right)
$$

ii) $\Lambda$ and $X$ are quasifinite and injective, respectively as a left $D$ comodule and a left $C$-comodule, and we have bicolinear isomorphisms

$$
\Lambda \cong{ }_{C} \mathrm{~h}(\mathrm{X}, \mathrm{C}) \text { and } \mathrm{U} \square_{\mathrm{C}} \mathrm{X} \square_{\mathrm{D}} \mathrm{V} \cong{ }_{\mathrm{D}} \mathrm{h}(\Lambda, \mathrm{D})
$$

Remark 4.4. If we take $U=C, V=C$ in Theorem 4.3, then we find the structure Theorem for Frobenius functors of the first kind between $\mathcal{M}^{C}$ and $\mathcal{M}^{D}$, see [12, Theorem 3.3].

\section{Comodules over Corings}

Let $A$ be a ring (with unit). An $A$-coring $\mathcal{C}$ is an $(A, A)$-bimodule together with two $(A, A)$-bimodule maps

$$
\Delta_{\mathcal{C}}: \mathcal{C} \rightarrow \mathcal{C} \otimes_{A} \mathcal{C} \text { and } \varepsilon_{\mathcal{C}}: \mathcal{C} \rightarrow A
$$


called a coproduct and a counit respectively, such that the usual coassociativity and counit properties hold, i.e.

$$
\begin{aligned}
\left(\Delta_{\mathcal{C}} \otimes_{A} I_{\mathcal{C}}\right) \circ \Delta_{\mathcal{C}} & =\left(I_{\mathcal{C}} \otimes_{A} \Delta_{\mathcal{C}}\right) \circ \Delta_{\mathcal{C}} \\
\left(\varepsilon_{\mathcal{C}} \otimes_{A} I_{\mathcal{C}}\right) \circ \Delta_{\mathcal{C}} & =\left(I_{\mathcal{C}} \otimes_{A} \varepsilon_{\mathcal{C}}\right) \circ \Delta_{\mathcal{C}}=I_{\mathcal{C}}
\end{aligned}
$$

Corings were first introduced by Sweedler in [28, and revived recently by Brzeziński in 2]. They can also be viewed as coalgebras in the monoidal category ${ }_{A} \mathcal{M}_{A}$.

A right $\mathcal{C}$-comodule is a right $A$-module $M$ together with a right $A$-module map $\rho^{r}: M \rightarrow M \otimes_{A} \mathcal{C}$ called a coaction such that

$$
\begin{aligned}
\left(\rho^{r} \otimes_{A} I_{\mathcal{C}}\right) \circ \rho^{r} & =\left(I_{M} \otimes_{A} \Delta_{\mathcal{C}}\right) \circ \rho^{r} \\
\left(I_{M} \otimes_{A} \varepsilon_{\mathcal{C}}\right) \circ \rho^{r} & =I_{M}
\end{aligned}
$$

In a similar way, we can define left $\mathcal{C}$-comodules and $(\mathcal{C}, \mathcal{C})$-bicomodules. We will use the Sweedler-Heyneman notation for corings and comodules over corings:

$$
\Delta_{\mathcal{C}}(c)=c_{(1)} \otimes_{A} c_{(2)} ; \rho^{r}(m)=m_{[0]} \otimes_{A} m_{[1]} \in M \otimes_{A} \mathcal{C}
$$

etc. A map $f: M \rightarrow N$ between (right) $\mathcal{C}$-comodules is called a $\mathcal{C}$-comodule map if $f$ is a right $A$-module map, and

$$
\rho^{r}(f(m))=f\left(m_{[0]}\right) \otimes_{A} m_{[1]}
$$

for all $m \in M . \mathcal{M}^{\mathcal{C}}$ is the category of right $\mathcal{C}$-comodules and $\mathcal{C}$-comodule maps. In a similar way, we introduce the categories

$$
{ }^{\mathcal{C}} \mathcal{M},{ }^{\mathcal{C}} \mathcal{M}^{\mathcal{C}},{ }_{A} \mathcal{M}^{\mathcal{C}}
$$

For example, ${ }_{A} \mathcal{M}^{\mathcal{C}}$ is the category of right $\mathcal{C}$-comodules that are also $(A, A)$ bimodules such that the right $\mathcal{C}$-comodule map is left $A$-linear. The morphisms between right $\mathcal{C}$-comodules are denoted by $\operatorname{Hom}^{\mathcal{C}}(\bullet, \bullet)$. Takeuchi has observed that entwined modules (see 4]) can be viewed as particular cases of comodules over a coring. A fortiori, the Doi-Koppinen Hopf modules ([13] and [20]) are special cases, and consequently Hopf modules, relative Hopf modules, graded modules, modules graded by $G$-sets and Yetter-Drinfel'd modules (see [13] and [9]), and we can apply the results we present below to all these types of modules.

Let $A$ be a ring, $\mathcal{C}$ an $A$-coring, and look at the forgetful functor

$$
F: \mathcal{M}^{\mathcal{C}} \rightarrow \mathcal{M}_{A} .
$$

Proposition 5.1. (2]) For an $A$-coring $\mathcal{C}$, the forgetful functor

$$
F: \mathcal{M}^{\mathcal{C}} \rightarrow \mathcal{M}_{A}
$$

has a right adjoint $G$. For $N \in \mathcal{M}_{A}, G(N)=N \otimes_{A} \mathcal{C}$, with structure induced by the structure on $\mathcal{C}$ :

$$
\left(n \otimes_{A} c\right) a=n \otimes_{A} c a \text { and } \rho^{r}\left(n \otimes_{A} c\right)=n \otimes_{A} c_{(1)} \otimes_{A} c_{(2)}
$$

For a morphism $f \in \mathcal{M}_{A}, G(f)=f \otimes_{A} I_{\mathcal{C}}$. 
Proof. We only give the unit and counit of the adjunction:

(16) $1_{\mathcal{M}^{\mathcal{C}}} \rightarrow G F \quad \eta_{M}=\rho^{r}: M \rightarrow M \otimes_{A} \mathcal{C} \quad \eta_{M}(m)=m_{[0]} \otimes_{A} m_{[1]}$

(k7:) $F G \rightarrow 1_{\mathcal{M}_{A}} \quad \varepsilon_{N}=I_{N} \otimes_{A} \varepsilon_{\mathcal{C}}: N \otimes_{A} \mathcal{C} \rightarrow N \quad \varepsilon_{N}\left(n \otimes_{A} c\right)=n \varepsilon_{\mathcal{C}}(c)$

We will investigate when $(F, G)$ is an $(\alpha, \beta)$-Frobenius pair, with $\alpha$ and $\beta$ of the following type:

$$
\begin{aligned}
& \alpha=I_{\mathcal{M}^{\mathcal{C}}}: \mathcal{M}^{\mathcal{C}} \rightarrow \mathcal{M}^{\mathcal{C}}: M \rightarrow M \\
& \beta=\bullet \otimes_{A} Q: \mathcal{M}_{A} \rightarrow \mathcal{M}_{A}, \quad \beta(N)=N \otimes_{A} Q
\end{aligned}
$$

where $Q$ is an $(A, A)$-bimodule.

As before, we need a description of $V=\underline{\underline{\operatorname{Nat}}}\left(G \beta F \alpha, 1_{\mathcal{M}^{\mathcal{C}}}\right)$ and $W=\underline{\underline{\operatorname{Nat}}}\left(1_{\mathcal{M}_{A}}, \beta F \alpha G\right)$.

First we need a Lemma.

Lemma 5.2. Take $\nu \in V$, and $N \in \mathcal{M}_{A}, G(N)=N \otimes_{A} \mathcal{C}$. Then

$$
\nu_{N \otimes_{A} \mathcal{C}}=I_{N} \otimes_{A} \nu_{\mathcal{C}}
$$

Proof. For $n \in N$, we consider $f_{n}: \mathcal{C} \rightarrow N \otimes_{A} \mathcal{C}, f_{n}(c)=n \otimes_{A} c . f_{n}$ is a morphism in $\mathcal{M}^{\mathcal{C}}$, and the naturality of $\nu$ produces a commutative diagram

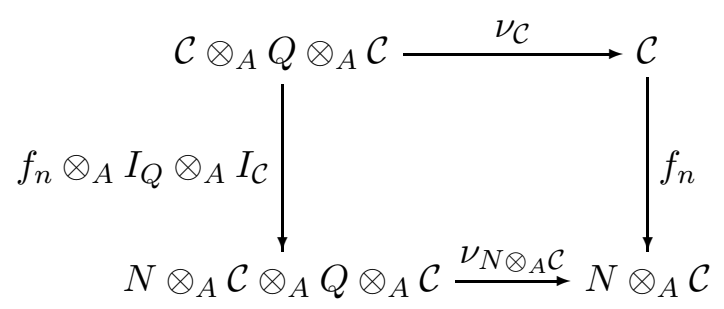

and we find

$$
\nu_{N \otimes_{A} \mathcal{C}}\left(n \otimes_{A} c \otimes_{A} q \otimes_{A} d\right)=n \otimes_{A} \nu_{\mathcal{C}}\left(c \otimes_{A} q \otimes_{A} d\right)
$$

Proposition 5.3. Let $\mathcal{C}$ be an $A$-coring and $V=\underline{\underline{\operatorname{Nat}}}\left(G \beta F, 1_{\mathcal{M}^{\mathcal{C}}}\right)$. We define

$$
V_{1}:={ }^{\mathcal{C}} \operatorname{Hom}^{\mathcal{C}}\left(\mathcal{C} \otimes_{\mathrm{A}} \mathrm{Q} \otimes_{\mathrm{A}} \mathcal{C}, \mathcal{C}\right)
$$

$V_{2}:=\left\{\theta \in{ }_{A} \operatorname{Hom}_{\mathrm{A}}\left(\mathcal{C} \otimes_{\mathrm{A}} \mathrm{Q} \otimes_{\mathrm{A}} \mathcal{C}, \mathrm{A}\right) \mid \mathrm{c}_{(1)} \theta\left(\mathrm{c}_{(2)} \otimes_{\mathrm{A}} \mathrm{q} \otimes_{\mathrm{A}} \mathrm{d}\right)=\theta\left(\mathrm{c} \otimes_{\mathrm{Aq}} \otimes_{\mathrm{A}} \mathrm{d}_{(1)}\right) \mathrm{d}_{(2)}\right.$ for all $\left.\mathrm{c}, \mathrm{d} \in \mathrm{C}, \mathrm{q} \in \mathrm{Q}\right\}$. Then

$$
V \cong V_{1} \cong V_{2}
$$

Proof. We define $\alpha: V \rightarrow V_{1}, \alpha(\nu)=\bar{\nu}=\nu_{\mathcal{C}}$. By definition, $\bar{\nu}$ is a morphism in $\mathcal{M}^{\mathcal{C}}$ and thus a right $\mathcal{C}$-comodule map. The properties on the left-hand side follow from the naturality of $\nu$ : for all $a \in A$, we consider the map $f_{a}: \mathcal{C} \rightarrow \mathcal{C}, f_{a}(c)=a c$, which is a morphism in $\mathcal{M}^{\mathcal{C}}$ since $\Delta_{\mathcal{C}}$ is 
an $(A, A)$-bimodule map, so that the naturality of $\nu$ gives a commutative diagram.

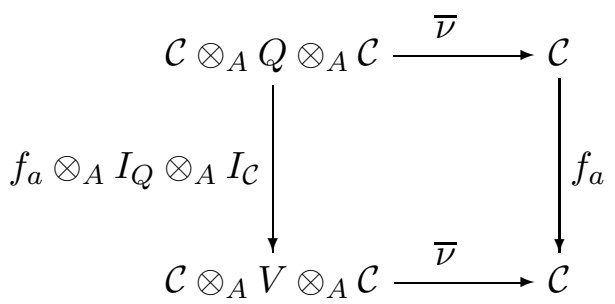

Applying the diagram to $c \otimes_{A} q \otimes_{A} d \in \mathcal{C} \otimes_{A} Q \otimes_{A} \mathcal{C}$, we find

$$
\bar{\nu}\left(a c \otimes_{A} q \otimes_{A} d\right)=a \bar{\nu}\left(c \otimes_{A} q \otimes_{A} d\right)
$$

and $\bar{\nu}$ is left $A$-linear.

The left $\mathcal{C}$-comodule structure map on $\mathcal{C} \otimes_{A} V \otimes_{A} \mathcal{C}$ is $\Delta_{\mathcal{C}} \otimes I_{V} \otimes I_{\mathcal{C}}$, and this map is a morphism in $\mathcal{M}^{\mathcal{C}}$, so that we have another commutative diagram

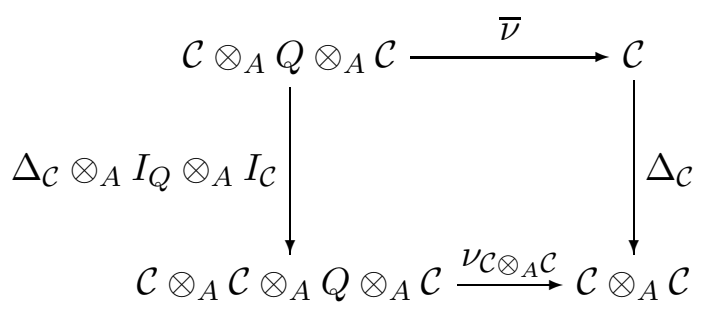

The above diagram tells us that $\bar{\nu}$ is a left $\mathcal{C}$-comodule map, and so, $\alpha$ is well-defined.

Next, we define $\alpha_{1}: V_{1} \rightarrow V_{2}$ :

$$
\alpha_{1}(\bar{\nu})=\theta=\varepsilon_{\mathcal{C}} \circ \bar{\nu}
$$

It is obvious that $\theta$ is an $(A, A)$-bimodule map. From the fact that $\bar{\nu}$ is a $\mathcal{C}$-bicomodule map, we find

$c_{(1)} \otimes_{A} \bar{\nu}\left(c_{(2)} \otimes_{A} q \otimes_{A} d\right)=\Delta_{\mathcal{C}}\left(\bar{\nu}\left(c \otimes_{A} q \otimes_{A} d\right)\right)=\bar{\nu}\left(c \otimes_{A} q \otimes d_{(1)}\right) \otimes_{A} d_{(2)}$

Applying $I_{\mathcal{C}} \otimes_{A} \varepsilon_{\mathcal{C}}$ to the first equality and $\varepsilon_{\mathcal{C}} \otimes_{A} I_{\mathcal{C}}$ to the second one, we find

$$
\bar{\nu}\left(c \otimes_{A} q \otimes_{A} d\right)=c_{(1)} \theta\left(c_{(2)} \otimes_{A} q \otimes_{A} d\right)=\theta\left(c \otimes_{A} q \otimes_{A} d_{(1)}\right) d_{(2)}
$$

and it follows that $\alpha_{1}$ is well-defined.

We define $\alpha_{1}^{-1}(\theta)=\bar{\nu}$ defined by (18). We will prove that $\bar{\nu}$ is a left $\mathcal{C}$ comodule map.

$$
\begin{aligned}
\left(I_{\mathcal{C}} \otimes_{A} \bar{\nu}\right) & { }^{l} \rho\left(c \otimes_{A} q \otimes_{A} d\right) \\
& =c_{(1)} \otimes_{A} \bar{\nu}\left(c_{(2)} \otimes_{A} q \otimes_{A} d\right) \\
& =c_{(1)} \otimes_{A} c_{(2)} \theta\left(c_{(3)} \otimes_{A} q \otimes_{A} d\right) \\
& =\Delta\left(c_{(1)}\right) \theta\left(c_{(2)} \otimes_{A} q \otimes_{A} d\right) \\
& =\Delta\left(c_{(1)} \theta\left(c_{(2)} \otimes_{A} q \otimes_{A} d\right)\right) \\
& ={ }^{l} \rho\left(\bar{\nu}\left(c \otimes_{A} q \otimes_{A} d\right)\right)
\end{aligned}
$$


Right $\mathcal{C}$-colinearity can be proved in the same way.

(18) also tells us that $\alpha_{1}^{-1}\left(\alpha_{1}(\bar{\nu})\right)=\bar{\nu}$. Conversely,

$$
\alpha_{1}\left(\alpha_{1}^{-1}(\theta)\right)\left(c \otimes_{A} q \otimes_{A} d\right)=\varepsilon_{\mathcal{C}}\left(c_{(1)}\right) \theta\left(c_{(2)} \otimes_{A} q \otimes_{A} d\right)=\theta\left(c \otimes_{A} q \otimes_{A} d\right)
$$

We still need to show that $\alpha$ is invertible. For $\bar{\nu} \in V_{1}$, and $\theta=\alpha_{1}(\bar{\nu})$, we define

by

$$
\nu=\alpha^{-1}(\bar{\nu}): G \beta F \alpha \rightarrow 1_{\mathcal{M}^{\mathcal{C}}}
$$

$\nu_{M}: M \otimes_{A} Q \otimes_{A} \mathcal{C} \rightarrow M ; \nu_{M}\left(m \otimes_{A} q \otimes_{A} c\right)=m_{[0]} \theta\left(m_{[1]} \otimes_{A} q \otimes_{A} c\right)$

$\nu$ is natural, since for every morphism $f: M \rightarrow M^{\prime}$ in $\mathcal{M}^{\mathcal{C}}$, we have that

$$
\begin{aligned}
\nu_{M^{\prime}}(f & \left.\otimes_{A} I_{Q} \otimes_{A} I_{\mathcal{C}}\right)\left(m \otimes_{A} q \otimes_{A} c\right) \\
& =\nu_{M^{\prime}}\left(f(m) \otimes_{A} q \otimes_{A} c\right)=f(m)_{[0]} \theta\left(f(m)_{[1]} \otimes_{A} q \otimes_{A} c\right) \\
& =f\left(m_{[0]}\right) \theta\left(m_{[1]} \otimes_{A} q \otimes_{A} c\right)=f\left(m_{[0]} \theta\left(m_{[1]} \otimes_{A} q \otimes_{A} c\right)\right) \\
& =f\left(\nu_{M}\left(m \otimes_{A} q \otimes_{A} c\right)\right)
\end{aligned}
$$

It is clear that $\alpha\left(\alpha^{-1}(\bar{\nu})\right)=\bar{\nu}$, since

$$
\nu_{\mathcal{C}}\left(c \otimes_{A} q \otimes_{A} d\right)=c_{(1)} \theta\left(c_{(2)} \otimes_{A} q \otimes_{A} d\right)=\bar{\nu}\left(c \otimes_{A} q \otimes_{A} d\right)
$$

Finally, let us show that $\alpha^{-1}(\alpha(\nu))=\nu$. The map $\rho^{r}: M \rightarrow M \otimes_{A} \mathcal{C}$ is in $\mathcal{M}^{\mathcal{C}}$. From Lemma 5.2, we know that $\nu_{M \otimes_{A} \mathcal{C}}=I_{M} \otimes_{A} \bar{\nu}$, so the naturality of $\nu$ generates a commutative diagram

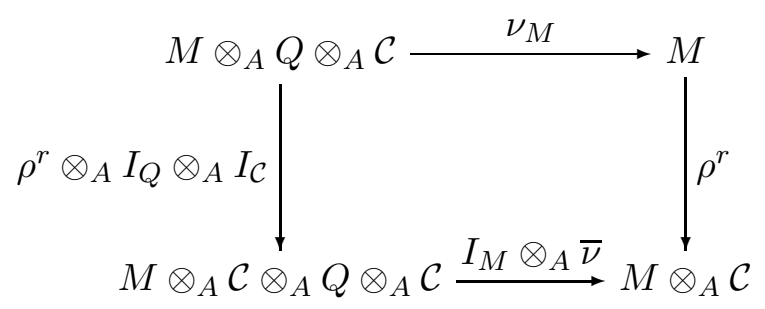

and we find

$$
\rho^{r}\left(\nu_{M}\left(m \otimes_{A} q \otimes_{A} c\right)\right)=m_{[0]} \otimes_{A} \bar{\nu}\left(m_{[1]} \otimes_{A} q \otimes_{A} c\right)
$$

Apply $\varepsilon_{\mathcal{C}}$ to the second factor:

$$
\nu_{M}\left(m \otimes_{A} q \otimes_{A} c\right)=m_{[0]} \theta\left(m_{[1]} \otimes_{A} q \otimes_{A} c\right)
$$

This means precisely that $\alpha^{-1}(\alpha(\nu))=\nu$.

Proposition 5.4. Let $\mathcal{C}$ be an $A$-coring and $W=\underline{\underline{\mathrm{Nat}}}\left(1_{\mathcal{M}_{A}}, \beta F \alpha G\right)$. We define

$$
W_{1}={ }_{A} \operatorname{Hom}_{\mathrm{A}}\left(\mathrm{A}, \mathcal{C} \otimes \otimes_{\mathrm{A}} \mathrm{Q}\right)
$$

and

$W_{2}=\left(\mathcal{C} \otimes_{A} V\right)^{A}=\left\{z=\sum z^{\mathcal{C}} \otimes_{A} z^{Q} \in \mathcal{C} \otimes_{A} Q \mid a \cdot\left(\sum z^{\mathcal{C}} \otimes_{A} z^{Q}\right)=\left(\sum z^{\mathcal{C}} \otimes_{A} z^{Q}\right) \cdot a\right.$, for all $\left.a \in A\right\}$.

Then

$$
W \cong W_{1} \cong W_{2} .
$$


Proof. We give the definitions of the connecting maps; other details are left to the reader.

$$
\begin{gathered}
\beta: W \rightarrow W_{1} ; \beta(\zeta)=\zeta_{A}=\bar{\zeta} \\
\beta_{1}: W_{1} \rightarrow W_{2} ; \beta_{1}(\bar{\zeta})=\bar{\zeta}(1) \\
\beta^{-1}: W_{1} \rightarrow W ; \beta^{-1}(\bar{\zeta})=\zeta
\end{gathered}
$$

with

$$
\zeta_{N}: N \rightarrow N \otimes_{A} \mathcal{C} \otimes_{A} V ; \zeta_{N}(n)=n \otimes_{A} \bar{\zeta}(1)
$$

We can prove now the main result of this section: for an $A$-coring $\mathcal{C}, F$ : $\mathcal{M}^{\mathcal{C}} \rightarrow \mathcal{M}_{A}$ will be the forgetful functor and $G=\bullet \otimes_{A} \mathcal{C}$ its right adjoint.

Theorem 5.5. Let $\mathcal{C}$ be an $A$-coring, $Q$ an $A$-bimodule and $\beta=\bullet \otimes_{A} Q$ : $\mathcal{M}_{A} \rightarrow \mathcal{M}_{A}$ the induction functor. The following statements are equivalent:

(1) $(F, G)$ is an $\left(I_{\mathcal{M}^{\mathcal{C}}}, \beta\right)$-Frobenius pair;

(2) There exists $\theta \in V_{2}$ and $z=\sum z^{\mathcal{C}} \otimes_{A} z^{Q} \in W_{2}$ such that

$$
\begin{aligned}
& \sum \theta\left(c \otimes_{A} q \otimes_{A} z^{\mathcal{C}}\right) z^{Q}=\varepsilon_{\mathcal{C}}(c) q \text { and } \sum \theta\left(z^{\mathcal{C}} \otimes_{A} z^{Q} \otimes_{A} c\right)=\varepsilon_{\mathcal{C}}(c) \\
& \text { for all } c \in \mathcal{C}, q \in Q .
\end{aligned}
$$

Proof. $G$ is also a left adjoint of $\beta F \alpha$ if and only if there exist natural transformations $\nu \in V$ and $\xi \in W$ such that

$$
\begin{aligned}
\beta F \alpha\left(\nu_{M}\right) \circ \xi_{\beta F \alpha} & =I_{\beta F \alpha} \\
\nu_{G(N)} \circ G\left(\xi_{N}\right) & =I_{G(N)}
\end{aligned}
$$

for all $M \in \mathcal{M}^{\mathcal{C}}$ and $N \in \mathcal{M}_{A}$. Now Propositions 5.3 and 5.4 give us the corresponding elements in $V_{2}$ and $W_{2}$. Now taking (20) with $M=\mathcal{C}$ and applying $\varepsilon_{\mathcal{C}}$ leads to

$$
\theta\left(c \otimes_{A} q \otimes_{A} z^{\mathcal{C}}\right) z^{Q}=\varepsilon_{\mathcal{C}}(c) q
$$

In a similar way, (21) with $N=A$ implies

$$
\sum \theta\left(z^{\mathcal{C}} \otimes_{A} z^{Q} \otimes_{A} c\right)=\varepsilon_{\mathcal{C}}(c)
$$

If we apply the above Theorem with $Q=A$ (i.e. $\beta=I_{\mathcal{M}_{A}}$ ), we can add one more equivalent condition to the four equivalent conditions given in [2, Theorem 4.1], without the assumption "C is projective over $A$ ".

Corollary 5.6. Let $\mathcal{C}$ be an $A$-coring. The following statements are equivalent:

(1) The forgetful functor $F: \mathcal{M}^{\mathcal{C}} \rightarrow \mathcal{M}_{A}$ is Frobenius (i.e. $(F, G)$ is a Frobenius pair of the first kind); 
(2) There exists a pair $(z, \theta)$, with $z \in \mathcal{C}^{A}$ and $\theta: \mathcal{C} \otimes_{A} \mathcal{C} \rightarrow A$ is an A-bimodule map such that

$$
\begin{aligned}
& c_{(1)} \theta\left(c_{(2)} \otimes_{A} d\right)=\theta\left(c \otimes_{A} d_{(1)}\right) d_{(2)}, \quad \theta\left(c \otimes_{A} z\right)=\theta\left(z \otimes_{A} c\right)=\varepsilon_{\mathcal{C}}(c) \\
& \quad \text { for all } c, d \in \mathcal{C} .
\end{aligned}
$$

Remark 5.7. As explained before, the categories of comodules over a coring generalize various kinds of Hopf modules. Moreover, the category of descent data asociated to a ring extension $S / R$ can be also viewed as the category of comodules over "the canonical coring" associated to the extension (see [8] for details). Therefore Theorem [5.5] can be applied in many situations. Perhaps, the most interesting application is the one where $Q=A_{\mu}$, where $\mu: A \rightarrow A$ is an automorphism of the ring $A$, i.e. the category equivalence $\beta$ is induced by a ring automorphism of $A$.

\section{REFERENCES}

[1] Bass, H. Algebraic K-theory: Benjamin: New York, 1968.

[2] Brzeziński, T. The structure of corings. Induction functors, Maschke-type theorem, and Frobenius and Galois properties. Algebras and Representation Theory 2002, 5, 389-414.

[3] Brzeziński, T.; Caenepeel, S.; Militaru, G.; Zhu, Shenglin. Frobenius and Maschke type Theorems for Doi-Hopf modules and entwined modules revisited: a unified approach. in Ring theory and Algebraic Geometry; Lecture Notes Pure Appl. Math.; Granja, A.; Hermida Alonso, J.; Verschoren, A.; eds.; Marcel Dekker: New York, 2001; vol. 221.

[4] Brzeziński, T.; Majid, S.; Coalgebra bundles. Comm. Math. Phys., 1998, 191 (2), 467-492.

[5] Caenepeel, S.; Ion, B.; Militaru, G. The structure of Frobenius algebras and separable algebras. K-theory 2000 (4), 19, 365-402.

[6] Caenepeel, S.; Ion, B.; Militaru, G.; Zhu, Shenglin. Separable functors for the category of Doi-Hopf modules, Applications. Adv. Math. 1999 (2), 145, 239-290.

[7] Caenepeel, S.; Kadison, L. Are biseparable extensions Frobenius? K-theory 2001, $24,361-383$.

[8] Caenepeel, S.; Militaru, G.; Zhu, Shenglin. Frobenius and separable functors for generalized module categories and nonlinear equations; Lecture Notes Math.; Springer Verlag: Berlin, 2002; vol. 1787.

[9] Caenepeel, S.; Militaru, G.; Zhu, Shenglin. Crossed modules and Doi-Hopf modules. Israel J. Math. 1997, 100, 221-247.

[10] Caenepeel, S.; Militaru, G.; Zhu, Shenglin. Doi-Hopf modules, Yetter-Drinfel'd modules and Frobenius type properties. Trans. Amer. Math. Soc. 1997, 349 (11), 43114342.

[11] Castaño Iglesias, F.; Gómez Torrecillas, J.; Năstăsescu, C. Separable functors in graded rings. J. Pure Appl. Algebra 1998, 127 (3), 219-230.

[12] Castaño Iglesias, F.; Gómez Torrecillas, J.; Năstăsescu, C. Frobenius functors. Applications. Comm. Algebra 1999, 27 (10), 4879-4900.

[13] Doi, Y. Unifying Hopf modules. J. Algebra 1992, 153 (2), 373-385.

[14] Kadison, L. The Jones polynomial and certain separable Frobenius extensions. J. Algebra 1996, 186 (2), 461-475.

[15] Kadison, L. Separability and the twisted Frobenius bimodules, Algebras and Representation Theory 1999, 2 (4), 397-414. 
[16] Kadison, L. New examples of Fobenius extensions; University Lect. Series; Amer. Math. Soc.: Providence, 1999; vol. 14.

[17] Kadison, L.; Stolin, A. An approach to Hopf algebras via Frobenius cooridinates II. Beiträge Algebra Geom. 2001, 42 (2), 359-384.

[18] Kasch, F. Grundlagen einer Theorie der Frobenius-Erweiterungen. Math. Ann. 1954, 127, 453-474.

[19] Kasch, F. Projektive Frobenius-Erweiterungen. Sitzungsber. Heidelberger Akad. Wiss., Math.-Naturw. Kl. 1961, 1960/61, 89-109.

[20] Koppinen, M. Variations on the smash product with applications to group-graded rings. J. Pure Appl. Algebra 1995, 104 (1), 61-80.

[21] Larson, R. G.; Sweedler, M. An associative bilinear form for Hopf algebras. Amer. J. Math. 1969, 91, 75-93.

[22] Mac Lane, S. Categories for the working mathematician, second edition; Graduate Texts in Mathematics; Springer-Verlag: New York, 1997; Vol. 5.

[23] Madar, A.; Marcus, A. Frobenius functors and transfer. Publ. Math. Debrecen, to appear.

[24] Morita, K.; Adjoint pairs of functors and Frobenius extensions. Sci. Rep. Tokyo Kyoiku Daigaku, Sect. A 1965, 9, 40-71.

[25] Nakayama, T.; Tsuzuku, T. On Frobenius extensions I. Nagoya Math. J. 1960, 17, 89-110.

[26] Pareigis, B. When Hopf algebras are Frobenius Algebras. J. Algebra 1971, 18, 588596.

[27] Pareigis, B. Endliche Hopf-Algebren; Vorlesungsarbeitung (Lecture Notes), Algebra Berichte; Verlag Uni-Druck - Verlag R. Fischer: München, 1973.

[28] Sweedler, M. The predual Theorem to the Jacobson-Bourbaki Theorem. Trans. Amer. Math. Soc. 1975, 213, 391-406.

[29] Sweedler, M. Hopf algebras; Benjamin: New York, 1969.

[30] Takeuchi, M. Morita Theorems for categories of comodules. J. Fac. Sci. Univ. Tokyo 1977, 24, 629-644.

Faculty of Engineering Sciences, Vrije Universiteit Brussel, VUB, B-1050 Brussels, Belgium

E-mail address: scaenepe@vub.ac.be

$U R L:$ http://homepages.vub.ac.be/ ${ }^{\sim}$ scaenepe/

Faculty of Engineering Sciences, Vrije Universiteit Brussel, VUB, B-1050

Brussels, Belgium

E-mail address: scaenepe@vub.ac.be

Faculty of Mathematics, University of Bucharest, Str. Academiei 14, RO70109 BuCHAREST 1, Romania

E-mail address: gmilit@al.math.unibuc.ro 